\title{
Inertial Iterative Schemes for D-Accretive Mappings in Banach Spaces and Curvature Systems
}

\author{
Li Wei ${ }^{1},{ }^{1}$ Wenwen Yue, ${ }^{1}$ Yingzi Shang, ${ }^{1}$ and Ravi P. Agarwal ${ }^{2}$ \\ ${ }^{1}$ School of Mathematics and Statistics, Hebei University of Economics and Business, Shijiazhuang 050061, Hebei, China \\ ${ }^{2}$ Department of Mathematics, Texas A \& M University-Kingsville, Kingsville, TX 78363, USA \\ Correspondence should be addressed to Li Wei; diandianba@yahoo.com
}

Received 30 December 2020; Revised 6 March 2021; Accepted 11 March 2021; Published 31 March 2021

Academic Editor: Xiaolong Qin

Copyright (c) $2021 \mathrm{Li}$ Wei et al. This is an open access article distributed under the Creative Commons Attribution License, which permits unrestricted use, distribution, and reproduction in any medium, provided the original work is properly cited.

\begin{abstract}
We propose and analyze a new iterative scheme with inertial items to approximate a common zero point of two countable $\mathrm{d}$-accretive mappings in the framework of a real uniformly smooth and uniformly convex Banach space. We prove some strong convergence theorems by employing some new techniques compared to the previous corresponding studies. We give some numerical examples to illustrate the effectiveness of the main iterative scheme and present an example of curvature systems to emphasize the importance of the study of d-accretive mappings.
\end{abstract}

\section{Introduction and Preliminaries}

In this paper, we assume that $E$ is a real Banach space and $E^{*}$ is the dual space of $E$. Suppose that $C$ is a nonempty closed and convex subset of $E$. The symbols " $\langle x, f\rangle$ ", “ $\rightarrow$ " and " - " denote the value of $f \in E^{*}$ at $x \in E$, the strong convergence, and the weak convergence either in $E$ or $E^{*}$, respectively.

The normalized duality mapping $J_{E}: E \rightarrow 2^{E^{*}}$ is defined as follows:

$$
J_{E}(x)=\left\{x^{*} \in E^{*}:\left\langle x, x^{*}\right\rangle=\|x\|^{2}=\left\|x^{*}\right\|^{2}\right\}, \quad \forall x \in E .
$$

Lemma 1 (see [1]). Assume that $E$ is real uniformly convex and uniformly smooth Banach space. Then (1) $J_{E}$ is singlevalued and surjective and, for $x \in E$ and $k \in(0,+\infty)$, $J_{E}(k x)=k J_{E}(x)$; (2) $J_{E}^{-1}=J_{E^{*}}$ is the normalized duality mapping from $E^{*}$ to $E$; (3) both $J_{E}$ and $J_{E}^{-1}$ are uniformly continuous on each bounded subset of $E$ or $E^{*}$, respectively.

Definition 1 (see [2]). The Lyapunov functional $\phi: E \times E \rightarrow R^{+}$is defined as follows:

$$
\phi(x, y)=\|x\|^{2}-2\left\langle x, j_{E}(y)\right\rangle+\|y\|^{2}, \quad \forall x, y \in E, j_{E}(y) \in J_{E}(y) .
$$

Similarly, the Lyapunov functional defined on $E^{*} \times E^{*}$ can be defined and denoted by $\bar{\phi}$.

Lemma 2 (see [3]). Let $E$ be a uniformly smooth and uniformly convex Banach space, and let $\left\{x_{n}\right\}$ and $\left\{y_{n}\right\}$ be two sequences in E. If either $\left\{x_{n}\right\}$ or $\left\{y_{n}\right\}$ is bounded and $\phi\left(x_{n}, y_{n}\right) \rightarrow 0$, as $n \rightarrow \infty$, then $x_{n}-y_{n} \rightarrow 0$, as $n \longrightarrow \infty$.

Definition 2 (see [4]). Let $\left\{C_{n}\right\}$ be a sequence of nonempty closed and convex subsets of $E$; then

(1) $s-\liminf C_{n}$, which is called strong lower limit of $\left\{C_{n}\right\}$, is defined as the set of all $x \in E$ such that there exists $x_{n} \in C_{n}$ for almost all $n$ and it tends to $x$ as $n \rightarrow \infty$ in the norm

(2) $w-\limsup C_{n}$, which is called weak upper limit of $\left\{C_{n}\right\}$, is defined as the set of all $x \in E$ such that there exists a subsequence $\left\{C_{n_{m}}\right\}$ of $\left\{C_{n}\right\}$ and $x_{n_{m}} \in C_{n_{m}}$ for every $n_{m}$ and it tends to $x$ as $n_{m} \rightarrow \infty$ in the weak topology 
(3) If $s-\liminf C_{n}=w-\limsup C_{n}$, then the common value is denoted by $\lim C_{n}$

Lemma 3 (see [4]). Let $\left\{C_{n}\right\}$ be a decreasing sequence of closed and convex subsets of $E$, that is, $C_{n} \subset C_{m}$, if $n \geq m$. Then, $\left\{C_{n}\right\}$ converges in $E$ and $\lim C_{n}=\cap_{n=1}^{\infty} C_{n}$.

Definition 3 (see $[1,2]$ ). Suppose that $E$ is a real uniformly smooth and uniformly convex Banach space and $C$ is a nonempty closed and convex subset of $E$; then, for each $x \in E$, there exists a unique element $v \in C$ such that $\|x-v\|=\inf \{\|x-y\|: y \in C\}$. Such an element $v$ is denoted by $P_{C} x$ and $P_{C}$ is called the metric projection of $E$ onto $C$.

Lemma 4 (see [5]). Suppose that $E$ is a real uniformly smooth and uniformly convex Banach space and $\left\{C_{n}\right\}$ is a sequence of nonempty closed and convex subsets of E. If $\lim _{n}$ exists and is not empty, then $\lim _{n \longrightarrow \infty} P_{C_{n}} x=P_{\lim C_{n}} x$, for $\forall x \in E$.

\section{Definition 4.}

(1) A mapping $T: E \longrightarrow E$ is said to be accretive [6] if $\left\langle T u_{1}-T u_{2}, J_{E}\left(u_{1}-u_{2}\right)\right\rangle \geq 0, \forall u_{i} \in E, i=1,2$

(2) A mapping T: $E \longrightarrow E$ is said to be d-accretive [7] if $\left\langle T u_{1}-T u_{2}, J_{E} u_{1}-J_{E} u_{2}\right\rangle \geq 0, \forall u_{i} \in E, i=1,2$

It is easy to see that accretive mappings and d-accretive mappings are identical in a Hilbert space, while they are different in a non-Hilbert space.

For a nonlinear mapping $A: D(A) \subset E \longrightarrow E$, we use $\operatorname{Fix}(A)=\{x \in D(A): A x=x\}$ and $A^{-1} 0=\{x \in D(A): A x=0\}$ to denote the fixed point set and zero point set of $A$, respectively.

Lemma 5 (see $[8,9])$. Suppose that $E$ is a real uniformly smooth and uniformly convex Banach space. Let $A: E \longrightarrow E$ be d-accretive mapping such that $R(I+A)=E$. Under the assumption that $A^{-1} 0 \neq \varnothing$, one has the following:

(1) $\forall x \in E^{*}, \forall z \in A^{-1} 0$, and $\forall r>0$,

$$
\begin{aligned}
& \bar{\phi}\left(J_{E} z,\left(J_{E^{*}}+r A J_{E^{*}}\right)^{-1} J_{E^{*}} x\right) \\
& \quad+\bar{\phi}\left(\left(J_{E^{*}}+r A J_{E^{*}}\right)^{-1} J_{E^{*}} x, x\right) \leq \bar{\phi}\left(J_{E} z, x\right) .
\end{aligned}
$$

(2) If $x_{n} \in E^{*}, x \in E^{*}, x_{n} \longrightarrow x$, and $\left(J_{E^{*}}+r A J_{E^{*}}\right)^{-1} J_{E^{*}}$ $x_{n} \longrightarrow x$, as $n \longrightarrow \infty$, then $x=\left(J_{E^{*}}+r A J_{E^{*}}\right)^{-1}$ $J_{E^{*}} x$.

Definition 5 (see [10]). Let $C$ be a nonempty closed subset of $E$ and let $Q$ be a mapping of $E$ onto $C$. Then $Q$ is said to be sunny if $Q(Q(x)+t(x-Q(x)))=Q(x)$, for all $x \in E$ and $t \geq 0$. A mapping $Q: E \longrightarrow C$ is said to be a retraction if $Q(z)=z$ for every $z \in C$. If $E$ is a smooth and strictly convex Banach space, then a sunny generalized nonexpansive retraction of $E$ onto $C$ is uniquely decided, which is denoted by $R_{C}$.
Definition 6 (see [3]). If $E$ is a real uniformly smooth and uniformly convex Banach space and $C$ is a nonempty closed and convex subset of $E$, then, for each $x \in E$, there exists a unique element $x_{0} \in C$ satisfying $\phi\left(x_{0}, x\right)=\inf \{\phi(z, x)$ $: z \in C\}$. In this case, $\forall x \in E$, define $\pi_{C}: E \longrightarrow C$ by $\pi_{C} x=x_{0}$, and $\pi_{C}$ is called the generalized projection from $E$ onto $C$.

Lemma 6 (see [11]). Suppose that $E$ is a real uniformly convex Banach space and $r \in(0,+\infty)$. Then there exists a continuous and strictly increasing function $g:[0,2 r] \longrightarrow[0,+\infty)$ with $g(0)=0$ satisfying

$$
\|\alpha x+(1-\alpha) y\|^{2} \leq \alpha\|x\|^{2}+(1-\alpha)\|y\|^{2}-\alpha(1-\alpha) g(\|x-y\|),
$$

for $\forall \alpha \in[0,1], \forall x, y \in E$ with $\|x\| \leq r$ and $\|y\| \leq r$.

Accretive mappings have been extensively studied until now and some works can be seen in [12-16] and the references therein. However, until 2000, some valuable research work has been done on d-accretive mappings. As we know, in 2000, Alber and Reich [17] presented the following iterative schemes for $\mathrm{d}$-accretive mapping $T$ in a real uniformly smooth and uniformly convex Banach space:

$$
\begin{aligned}
& x_{n+1}=x_{n}-\alpha_{n} T x_{n}, \\
& x_{n+1}=x_{n}-\alpha_{n} \frac{T x_{n}}{\left\|T x_{n}\right\|}, \quad n \geq 0 .
\end{aligned}
$$

They proved that the iterative sequences $\left\{x_{n}\right\}$ generated by (5) and (6) converge weakly to the zero point of $T$ under the assumption that $T$ is uniformly bounded and demicontinuous.

In 2006, Guan [18] presented the following projective method for the $d$-accretive mapping $T$ in a real uniformly smooth and uniformly convex Banach space $E$ :

$$
\left\{\begin{array}{l}
x_{1} \in D(T), \\
y_{n}=\left(I+r_{n} T\right)^{-1} x_{n}, \\
C_{n}=\left\{v \in D(T): \phi\left(v, y_{n}\right) \leq \phi\left(v, x_{n}\right)\right\}, \\
Q_{n}=\left\{v \in D(T):\left\langle x_{n}-v, J_{E} x_{1}-J_{E} x_{n}\right\rangle \geq 0\right\}, \\
x_{n+1}=\pi_{C_{n} \cap Q_{n}} x_{1}, \quad n \geq 1 .
\end{array}\right.
$$

It was shown that the iterative sequences $\left\{x_{n}\right\}$ generated by (7) converge strongly to the zero point of $T$ under the assumptions that (1) $R(I+T)=E, \quad$ (2) $T$ is demicontinuous, and (3) $J_{E}$ is weakly sequentially continuous and satisfies

$$
\phi\left(p,\left(I+r_{n} T\right)^{-1} x\right) \leq \phi(p, x),
$$

for $\forall x \in E$ and $p \in T^{-1} 0$. The restrictions are extremely strong, and it is hard for us to find such a $d$-accretive mapping that is demicontinuous and satisfies (8).

In 2014, Wei et al. [7] presented the following block iterative schemes for approximating common zero points of $d$-accretive mappings $\left\{T_{i}\right\}_{i=1}^{m}$ in a Banach space $E$ : 


$$
\left\{\begin{array}{l}
x_{1} \in E \\
y_{n}=\sum_{i=1}^{m} \omega_{n, i}\left[\alpha_{n, i} x_{n}+\left(1-\alpha_{n, i}\right)\left(I+r_{n, i} T_{i}\right)^{-1} x_{n}\right], \\
x_{n+1}=\sum_{i=1}^{m} \eta_{n, i}\left[\beta_{n, i} x_{n}+\left(1-\beta_{n, i}\right)\left(I+s_{n, i} T_{i}\right)^{-1} y_{n}\right], n \in N,
\end{array}\right.
$$

$$
\left\{\begin{array}{l}
x_{1} \in E, \\
u_{n}=\sum_{i=1}^{m} \omega_{n, i}\left[\alpha_{n, i} x_{n}+\left(1-\alpha_{n, i}\right)\left(I+r_{n, i} T_{i}\right)^{-1} x_{n}\right], \\
v_{n+1}=\sum_{i=1}^{m} \eta_{n, i}\left[\beta_{n, i} x_{n}+\left(1-\beta_{n, i}\right)\left(I+s_{n, i} T_{i}\right)^{-1} u_{n}\right], \\
H_{1}=E, \\
H_{n+1}=\left\{z \in H_{n}: \phi\left(u_{n}, z\right) \leq \phi\left(x_{n}, z\right)\right\}, \\
x_{n+1}=R_{H_{n+1}} x_{1}, \quad n \in N .
\end{array}\right.
$$

Under mild assumptions, $\left\{x_{n}\right\}$ generated by (9) is proved to be weakly convergent to an element in $\cap_{i=1}^{m} T_{i}^{-1} 0$, while (10) is proved to be strongly convergent to an element in $\cap_{i=1}^{m} T_{i}^{-1} 0$.
In [19], the study on finite d-accretive mappings is extended to that for infinite $\mathrm{d}$-accretive mappings $\left\{T_{i}\right\}_{i=1}^{\infty} \subset E \times E$ :

$$
\left\{\begin{array}{l}
u_{1}=v \in E^{*}, \\
w_{n, i}=\left(I+s_{n, i} J_{E} T_{i} J_{E^{*}}\right)^{-1} u_{n}, \\
U_{1}=E^{*} \\
U_{n+1, i}=\left\{z \in E^{*}:\left\langle J_{E^{*}}\left(u_{n}-w_{n, i}\right), w_{n, i}-z\right\rangle \geq 0\right\}, \\
U_{n+1}=\left(\bigcap_{i=1}^{\infty} U_{n+1, i}\right) \cap U_{n}, \\
V_{n+1}=\left\{z \in U_{n+1}:\|v-z\|^{2} \leq\left\|P_{U_{n+1}}(v)-v\right\|^{2}+\tau_{n+1}\right\} \\
u_{n+1} \in V_{n+1}, \\
\frac{u_{n}}{n}=J_{E^{*}} u_{n}, \quad n \in N .
\end{array}\right.
$$

Then, sequence $\left\{\bar{u}_{n}\right\}$ generated by $(11)$ is proved to be strongly convergent to an element in $\cap_{i=1}^{m} T_{i}^{-1} 0$.

A new idea can be seen in (11), where the iterative element $u_{n} \in V_{n}$ can be chosen arbitrarily, which is different from the traditional one, for example, (7) in [18]. However, it is found that, for each iterative step $n$ in (11), countable sets $U_{n+1, i}$ should be evaluated for $i \in N$. To simplify it theoretically, the following iterative scheme is designed in [9]:

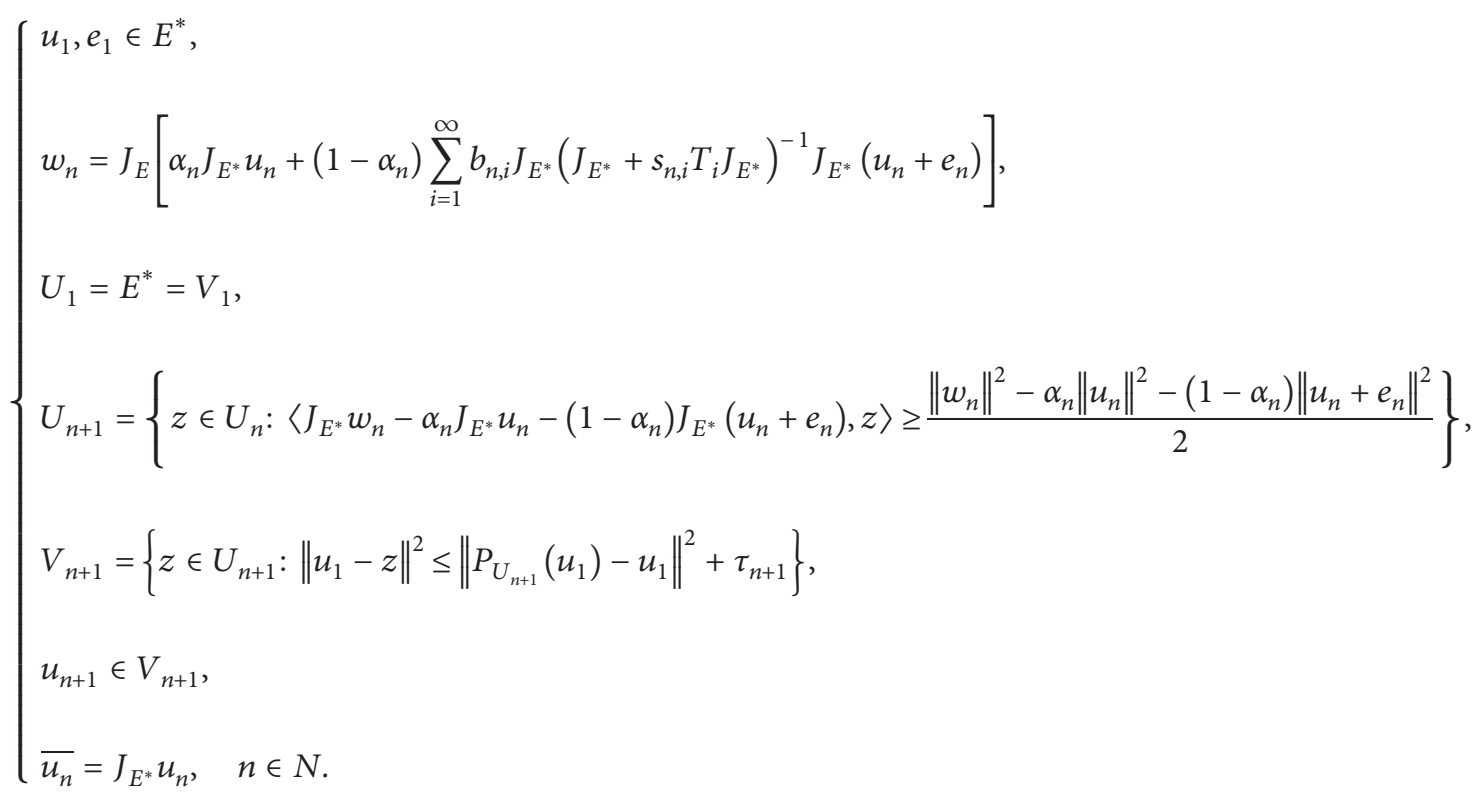

Then, $\left\{\overline{u_{n}}\right\}$ generated by (12) is proved to be strongly convergent to an element in $\cap_{i=1}^{m} T_{i}^{-1} 0$.

In 2020, Wei et al. [8] extended the discussion on countable d-accretive mappings $\left\{T_{i}\right\}_{i=1}^{\infty}$ to that for two groups of countable d-accretive mappings $\left\{T_{i}\right\}_{i=1}^{\infty}$ and $\left\{S_{i}\right\}_{i=1}^{\infty}$ and construct two key groups of sets $\left\{V_{n}\right\}$ and $\left\{Y_{n}\right\}$, where the iterative elements $\left\{y_{n}\right\}$ and $\left\{u_{n}\right\}$ can be chosen arbitrarily in $\left\{V_{n}\right\}$ and $\left\{Y_{n}\right\}$, respectively. 


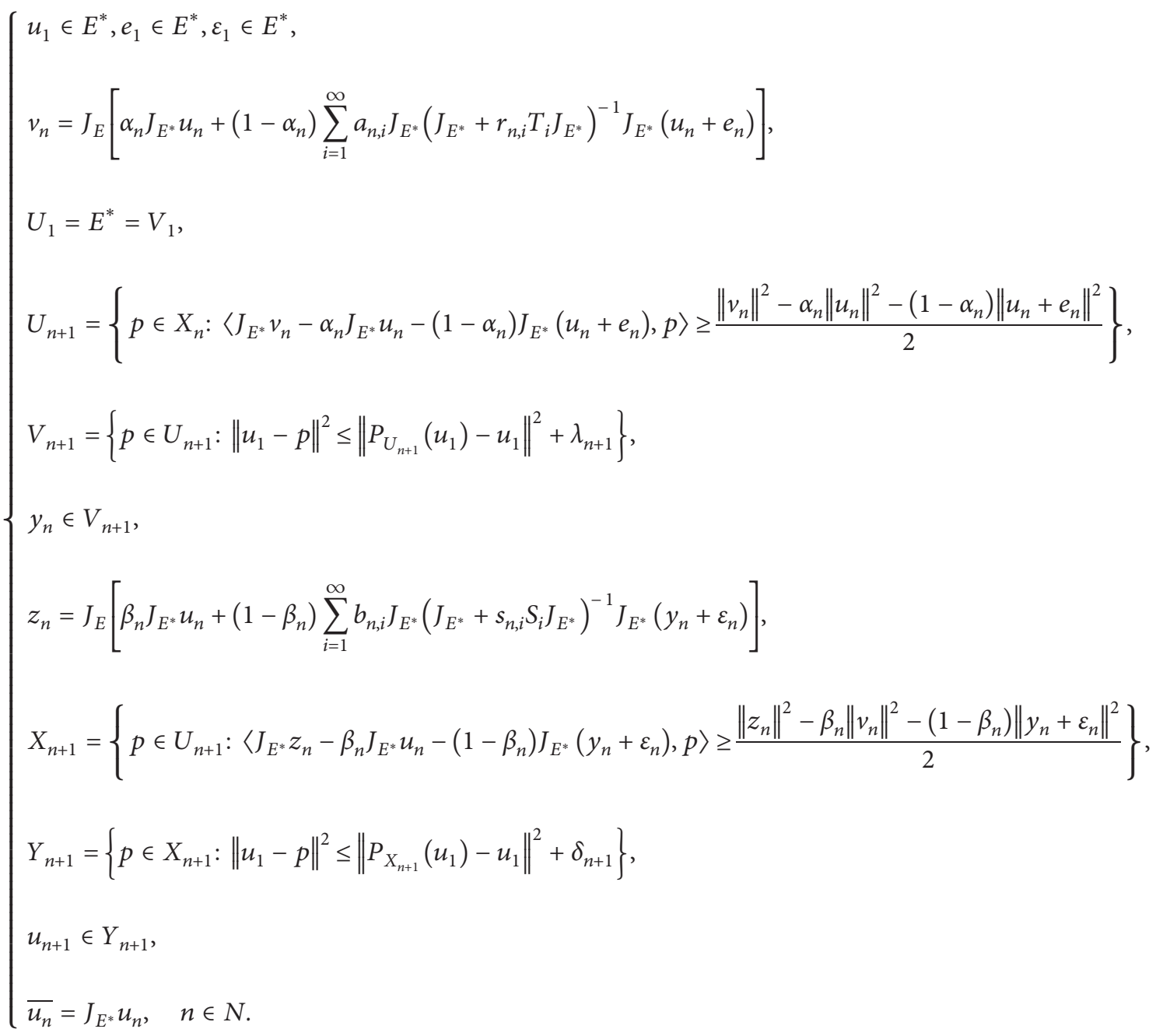

Then $\left\{\overline{u_{n}}\right\}$ generated by (13) is proved to be strongly convergent to an element in $\left(\cap_{i=1}^{\infty} T_{i}^{-1} 0\right) \cap\left(\cap_{i=1}^{\infty} S_{i}^{-1} 0\right)$.

Recall that the inertial-type algorithm was first proposed by Polyak [20] as an acceleration process in solving a smooth convex minimization problem. The inertial-type algorithm involves a two-step iterative method where the next iterate is defined by making use of the previous two iterates. For example, in 2015, Lorenz and Pock [21] proposed the following inertial forwardbackward algorithm for approximating zero points of $T+S$, where $T$ and $S$ are accretive-type mappings in Hilbert space $H$ :

$$
\left\{\begin{array}{l}
u_{0}, u_{1} \in H \text { chosen arbitrarily, } \\
v_{n}=u_{n}+\theta_{n}\left(u_{n}-u_{n-1}\right), \\
u_{n+1}=\left(I+r_{n} T\right)^{-1}\left(v_{n}-r_{n} S v_{n}\right), \quad n \in N .
\end{array}\right.
$$

In (14), the term $\theta_{n}\left(u_{n}-u_{n-1}\right)$ is called the inertial term.

In this paper, motivated by the previous work, some new work is done in the construction of new iterative schemes: (i) the inertial term is inserted for the purpose of possible acceleration; (ii) the combination expressions of $T_{i}$ or $S_{i}$ are different from those in (11)-(13). Numerical experiments are conducted, and it is very interesting that the rate of convergence is so quick that only eight steps are enough for some special cases and for different choices of iterative elements. To emphasize the importance of the topic, a kind of curvature systems is studied and is taken as an example of $\mathrm{d}$-accretive mappings.

\section{Iterative Schemes and Strong Convergence Theorems}

2.1. Iterative Schemes. In this section, we suppose that the following conditions are satisfied:

$\left(A_{1}\right) E$ is a real uniformly convex and uniformly smooth Banach space; $J_{E}: E \longrightarrow E^{*}$ and $J_{E^{*}}: E^{*} \longrightarrow E$ are the normalized duality mappings.

$\left(A_{2}\right) T_{i}, S_{i}: E \longrightarrow E$ are d-accretive mappings such that $R\left(I+T_{i}\right)=E=R\left(I+S_{i}\right)$, for each $i \in N$.

$\left(A_{3}\right)\left\{r_{n, i}\right\},\left\{t_{n, i}\right\},\left\{\xi_{n}\right\}$ and $\left\{\tau_{n}\right\}$ are real number sequences in $(0,+\infty)$, for $i, n \in N .\left\{\lambda_{n}\right\}$ and $\left\{\vartheta_{n}\right\}$ are real number sequences in $(-\infty,+\infty) .\left\{\alpha_{n}\right\}_{n=1}^{\infty}$ and $\left\{\beta_{n}\right\}_{n=1}^{\infty}$ are real number sequences in $[0,1]$. 
$\left(A_{4}\right) \quad a_{0}, b_{0} \in(0,1), \quad\left\{a_{n, i}\right\}_{i=0}^{\infty}$ and $\left\{b_{n, i}\right\}_{i=0}^{\infty}$ are real number sequences in $(0,1)$ such that $a_{0}+\sum_{i=1}^{\infty} a_{n, i}=b_{0}+\sum_{i=1}^{\infty} b_{n, i}=1$.

$\left(A_{5}\right)\left\{\varepsilon_{n}^{(1)}\right\}$ and $\left\{\varepsilon_{n}^{(2)}\right\}$ are the error sequences in $E^{*}$.

$\left(\mathrm{A}_{6}\right)$

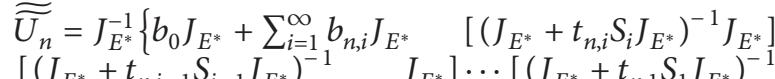
$\left.\left.J_{E^{*}}\right]\right\}$.

We construct the following iterative scheme:

$$
\left\{\begin{array}{l}
x_{0}, x_{1}, \varepsilon_{1}^{(1)}, \varepsilon_{1}^{(2)} \in E^{*}, \\
u_{n}=x_{n}+\lambda_{n}\left(x_{n}-x_{n-1}\right), \\
z_{n}=x_{n}+\vartheta_{n}\left(x_{n}-x_{n-1}\right), \\
v_{n}=J_{E^{*}}^{-1}\left[\left(1-\alpha_{n}\right) J_{E^{*}} u_{n}+\alpha_{n} J_{E^{*}} \widetilde{U_{n}}\left(z_{n}+\varepsilon_{n}^{(1)}\right)\right], \\
V_{1}=W_{1}=E^{*}, \\
V_{n+1}=\left\{p \in V_{n}: \bar{\phi}\left(p, v_{n}\right) \leq\left(1-\alpha_{n}\right) \bar{\phi}\left(p, u_{n}\right)+\alpha_{n} \bar{\phi}\left(p, z_{n}+\varepsilon_{n}^{(1)}\right)\right\}, \\
W_{n+1}=\left\{p \in V_{n+1}:\left\|x_{1}-p\right\|^{2} \leq\left\|P_{V_{n+1}}\left(x_{1}\right)-x_{1}\right\|^{2}+\tau_{n+1}\right\}, \\
y_{n} \in W_{n+1} \text { chosen arbitrarily, } \\
w_{n}=J_{E^{*}}^{-1}\left[\left(1-\beta_{n}\right) J_{E^{*}} u_{n}+\beta_{n} J_{E^{*}} \widetilde{U_{n}}\left(y_{n}+\varepsilon_{n}^{(2)}\right)\right], \\
U_{1}=X_{1}=E^{*}, \\
U_{n+1}=\left\{p \in V_{n+1}: \bar{\phi}\left(p, w_{n}\right) \leq\left(1-\beta_{n}\right) \bar{\phi}\left(p, u_{n}\right)+\beta_{n} \bar{\phi}\left(p, y_{n}+\varepsilon_{n}^{(2)}\right)\right\}, \\
X_{n+1}=\left\{p \in U_{n+1}:\left\|x_{1}-p\right\|^{2} \leq\left\|P_{U_{n+1}}\left(x_{1}\right)-x_{1}\right\|^{2}+\xi_{n+1}\right\}, \\
x_{n+1} \in X_{n+1} \text { chosen arbitrarily, } n \in N .
\end{array}\right.
$$

\subsection{Strong Convergence Theorems}

Theorem 1. Consider $\left(\cap_{i=1}^{\infty} T_{i}^{-1} 0\right) \cap\left(\cap_{i=1}^{\infty} S_{i}^{-1} 0\right) \neq \varnothing$, inf $_{n}$ $r_{n, i}>0, \inf _{n} t_{n, i}>0$ for $i \in N, \alpha_{n} \nrightarrow 0, \beta_{n} \nrightarrow 0, \tau_{n} \longrightarrow 0, \lambda_{n}$ $\nrightarrow \pm \infty, \vartheta_{n} \nrightarrow \pm \infty, \xi_{n} \longrightarrow 0, \tau_{n} \longrightarrow 0, \varepsilon_{n}^{(1)} \longrightarrow 0$, and $\varepsilon_{n}^{(2)} \longrightarrow 0$, as $n \longrightarrow \infty$. Then, the iterative sequence $\overline{x_{n}}=J_{E^{*}}$ $x_{n} \longrightarrow J_{E^{*}} \quad P_{\cap_{n=1}^{\infty} U_{n}}\left(x_{1}\right)=J_{E^{*}} P_{\left(\cap_{i=1}^{\infty}\left(T_{i} J_{E^{*}}\right)^{-1} 0\right) \cap\left(\cap_{i=1}^{\infty}\left(S_{i} J_{E^{*}}\right)^{-1} 0\right)}$ $\in\left(\cap_{i=1}^{\infty} T_{i}^{-1} 0\right) \cap\left(\cap_{i=1}^{\infty} S_{i}^{-1} 0\right)$, as $n \longrightarrow \infty$.

Proof. The proof is split into eight steps.

Step 1. $\cap_{n=1}^{\infty} U_{n} \neq \varnothing$.
Since $\left(\cap_{i=1}^{\infty} T_{i}^{-1} 0\right) \cap\left(\cap_{i=1}^{\infty} S_{i}^{-1} 0\right) \neq \varnothing$, there exists $\delta_{0} \in E$ such that $T_{i} \delta_{0}=S_{i} \delta_{0}=0, \forall i \in N$. It follows from Lemma 1 that there exists $\eta_{0} \in E^{*}$ such that $J_{E^{*}} \eta_{0}=\delta_{0} . \quad$ Therefore, $\quad\left(\cap_{i=1}^{\infty}\left(T_{i} J_{E^{*}}\right)^{-1} 0\right) \cap$ $\left(\cap_{i=1}^{\infty}\left(S_{i} J_{E^{*}}\right)^{-1} 0\right) \neq \varnothing$.

Next, we shall use inductive method to prove that $\left(\cap_{i=1}^{\infty}\left(T_{i} J_{E^{*}}\right)^{-1} 0\right) \cap\left(\cap_{i=1}^{\infty}\left(S_{i} J_{E^{*}}\right)^{-1} 0\right) \subset U_{n}, n \in N$.

$\forall p \in\left(\cap_{i=1}^{\infty}\left(T_{i} J_{E^{*}}\right)^{-1} 0\right) \cap\left(\cap_{i=1}^{\infty}\left(S_{i} J_{E^{*}}\right)^{-1} 0\right)$. For $n=1$, it is obvious that $p \in U_{1}$. Suppose that the result is true for $n=k$. Then, if $n=k+1$, it follows from the definition of the Lyapunov functional, the convexity of $\|\cdot\|^{2}$, and Lemma 5 that 


$$
\begin{aligned}
\bar{\phi}\left(p, v_{k}\right)= & \|p\|^{2}-2\left(1-\alpha_{k}\right)\left\langle p, J_{E^{*}} u_{k}\right\rangle-2 \alpha_{k}\left\langle p, J_{E^{*}} \widetilde{U_{k}}\left(z_{k}+\varepsilon_{k}^{(1)}\right)\right\rangle \\
& +\left\|\left(1-\alpha_{k}\right) J_{E^{*}} u_{k}+\alpha_{k} J_{E^{*}} \widetilde{U_{k}}\left(z_{k}+\varepsilon_{k}^{(1)}\right)\right\|^{2} \\
& \leq\|p\|^{2}-2\left(1-\alpha_{k}\right)\left\langle p, J_{E^{*}} u_{k}\right\rangle-2 \alpha_{k} a_{0}\left\langle p, J_{E^{*}}\left(z_{k}+\varepsilon_{k}^{(1)}\right)\right\rangle-2 \alpha_{k}\left\langle p, \sum_{i=1}^{\infty} a_{k, i} J_{E^{*}}\left(J_{E^{*}}+r_{k, i} T_{i} J_{E^{*}}\right)^{-1} J_{E^{*}}\left(z_{k}+\varepsilon_{k}^{(1)}\right)\right\rangle \\
& +\left(1-\alpha_{k}\right)\left\|u_{k}\right\|^{2}+\alpha_{k} a_{0}\left\|z_{k}+\varepsilon_{k}^{(1)}\right\|^{2}+\alpha_{k} \sum_{i=1}^{\infty} a_{k, i}\left\|\left(J_{E^{*}}+r_{k, i} T_{i} J_{E^{*}}\right)^{-1} J_{E^{*}}\left(z_{k}+\varepsilon_{k}^{(1)}\right)\right\|^{2} \\
= & \left(1-\alpha_{k}\right) \bar{\phi}\left(p, u_{k}\right)+\alpha_{k} a_{0} \bar{\phi}\left(p, z_{k}+\varepsilon_{k}^{(1)}\right)+\alpha_{k} \sum_{i=1}^{\infty} a_{k, i} \bar{\phi}\left(p,\left(J_{E^{*}}+r_{k, i} T_{i} J_{E^{*}}\right)^{-1} J_{E^{*}}\left(z_{k}+\varepsilon_{k}^{(1)}\right)\right) \\
& \leq\left(1-\alpha_{k}\right) \bar{\phi}\left(p, u_{k}\right)+\alpha_{k} \bar{\phi}\left(p, z_{k}+\varepsilon_{k}^{(1)}\right) .
\end{aligned}
$$

Thus, $p \in V_{k+1}$. Using Lemma 5 repeatedly, similar to the above discussion, one has

$$
\begin{aligned}
\bar{\phi} & \left(p, w_{k}\right) \leq\left(1-\beta_{k}\right) \bar{\phi}\left(p, u_{k}\right)+\beta_{k} \bar{\phi}\left(p, \widetilde{\widetilde{U_{k}}}\left(y_{k}+\varepsilon_{k}^{(2)}\right)\right) \\
\leq & \left(1-\beta_{k}\right) \bar{\phi}\left(p, u_{k}\right) \\
& +\beta_{k} b_{0} \bar{\phi}\left(p, y_{k}+\varepsilon_{k}^{(2)}\right)+\beta_{k} \sum_{i=1}^{\infty} b_{k, i} \bar{\phi}\left(p, y_{k}+\varepsilon_{k}^{(2)}\right) \\
= & \left(1-\beta_{k}\right) \bar{\phi}\left(p, u_{k}\right)+\beta_{k} \bar{\phi}\left(p, y_{k}+\varepsilon_{k}^{(2)}\right) .
\end{aligned}
$$

Then $p \in U_{k+1}$, which implies that $\cap_{n=1}^{\infty} U_{n} \neq \varnothing$.

Step 2. Both $V_{n}$ and $U_{n}$ are closed and convex subsets of $E^{*}$, for $n \in N$.

If $n=1$, the result is obvious. If $n \geq 2$, since

$$
\begin{aligned}
\bar{\phi}\left(p, v_{n}\right) & \leq\left(1-\alpha_{n}\right) \bar{\phi}\left(p, u_{n}\right)+\alpha_{n} \bar{\phi}\left(p, z_{n}+\varepsilon_{n}^{(1)}\right) \\
& \Leftrightarrow\left\langle p,\left(1-\alpha_{n}\right) J_{E^{*}} u_{n}+\alpha_{n} J_{E^{*}}\left(z_{n}+\varepsilon_{n}^{(1)}\right)-J_{E^{*}} v_{n}\right\rangle \leq \frac{\alpha_{n}\left\|z_{n}+\varepsilon_{n}^{(1)}\right\|^{2}+\left(1-\alpha_{n}\right)\left\|u_{n}\right\|^{2}-\left\|v_{n}\right\|^{2}}{2} .
\end{aligned}
$$

$V_{n}$ is a closed and convex subset of $E^{*}$, for $n \in N$.

Since

$$
\begin{aligned}
\bar{\phi}\left(p, w_{n}\right) & \leq\left(1-\beta_{n}\right) \bar{\phi}\left(p, u_{n}\right)+\beta_{n} \bar{\phi}\left(p, y_{n}+\varepsilon_{n}^{(2)}\right) \\
& \Leftrightarrow\left\langle p,\left(1-\beta_{n}\right) J_{E^{*}} u_{n}+\beta_{n} J_{E^{*}}\left(y_{n}+\varepsilon_{n}^{(2)}\right)-J_{E^{*}} w_{n}\right\rangle \leq \frac{\beta_{n}\left\|y_{n}+\varepsilon_{n}^{(2)}\right\|^{2}+\left(1-\beta_{n}\right)\left\|u_{n}\right\|^{2}-\left\|w_{n}\right\|^{2}}{2} .
\end{aligned}
$$

$U_{n}$ is a closed and convex subset of $E^{*}$, for $n \in N$.

Step 3. $P_{U_{n}}\left(x_{1}\right) \longrightarrow P_{\cap_{n=1}^{\infty} U_{n}}\left(x_{1}\right)$, as $n \longrightarrow \infty$.

The result follows from the results of Steps 1 and 2 and Lemmas 3 and 4.

Step $4 . W_{n} \neq \varnothing$ and $X_{n} \neq \varnothing$.

Since $\left\|P_{V_{n+1}}\left(x_{1}\right)-x_{1}\right\|=\inf _{q \in V_{n+1}}\left\|q-x_{1}\right\|$; then, for $\tau_{n+1}$, there exists $\theta_{n+1} \in V_{n+1}$ such that

$$
\begin{aligned}
& \left\|x_{1}-\theta_{n+1}\right\|^{2} \leq\left(\inf _{q \in V_{n+1}}\left\|q-x_{1}\right\|\right)^{2}+\tau_{n+1} \\
& =\left\|P_{V_{n+1}}\left(x_{1}\right)-x_{1}\right\|^{2}+\tau_{n+1} .
\end{aligned}
$$

Then $W_{n} \neq \varnothing$. Similarly, $X_{n} \neq \varnothing$. This ensures that $\left\{x_{n}\right\}$ is well defined.

Step 5. $x_{n} \longrightarrow P_{\cap_{n=1}^{\infty} U_{n}}\left(x_{1}\right)$, as $n \longrightarrow \infty$. 
Since

$\left\|x_{1}-x_{n+1}\right\|^{2} \leq\left\|P_{U_{n+1}}\left(x_{1}\right)-x_{1}\right\|^{2}+\xi_{n+1}$. It follows from Step 3 and $\xi_{n} \longrightarrow 0$ that $\left\{x_{n}\right\}$ is bounded.
Since $x_{n+1} \in X_{n+1} \subset U_{n+1}$ and $U_{n}$ is convex, for $\forall t \in(0,1), \quad t P_{U_{n+1}}\left(x_{1}\right)+(1-t) x_{n+1} \in U_{n+1}$. Using Lemma 6, one has

$$
\begin{aligned}
\left\|P_{U_{n+1}}\left(x_{1}\right)-x_{1}\right\|^{2} & \leq\left\|t P_{U_{n+1}}\left(x_{1}\right)+(1-t) x_{n+1}-x_{1}\right\|^{2} \\
& \leq t\left\|P_{U_{n+1}}\left(x_{1}\right)-x_{1}\right\|^{2}+(1-t)\left\|x_{n+1}-x_{1}\right\|^{2}-t(1-t) g\left(\left\|P_{U_{n+1}}\left(x_{1}\right)-x_{n+1}\right\|\right) .
\end{aligned}
$$

Therefore, $\operatorname{tg}\left(\left\|P_{U_{n+1}}\left(x_{1}\right)-x_{n+1}\right\|\right) \leq\left\|x_{n} \quad+1-x_{1}\right\|^{2}-$ $\left\|P_{U_{n+1}}\left(x_{1}\right)-x_{1}\right\|^{2} \leq \xi_{n+1}^{n+1} \longrightarrow 0$, as $n \longrightarrow \infty$. Therefore, $x_{n+1}-P_{U_{n+1}}\left(x_{1}\right) \longrightarrow \infty$, as $n \longrightarrow \infty$. Combining with Step $3, x_{n} \longrightarrow P_{\cap_{n=1}^{\infty} U_{n}}\left(x_{1}\right), n \longrightarrow \infty$.

Step 6. $u_{n} \longrightarrow P_{\bigcap_{n=1}^{\infty} U_{n}}\left(x_{1}\right), z_{n} \longrightarrow P_{\cap_{n=1}^{\infty} U_{n}}\left(x_{1}\right), v_{n} \longrightarrow$ $P_{\cap_{n=1}^{\infty} U_{n}}\left(x_{1}\right)$, and $y_{n} \longrightarrow P_{\cap_{n=1}^{\infty} U_{n}}\left(x_{1}\right)$, as $n \longrightarrow \infty$.
In fact, since $u_{n}=x_{n}+\lambda_{n}\left(x_{n}-x_{n-1}\right)$ with $\lambda_{n} \rightarrow \pm \infty$, $u_{n} \longrightarrow P_{\cap_{n=1}^{\infty} U_{n}}\left(x_{1}\right)$, as $n \longrightarrow \infty$. Similarly, $z_{n} \longrightarrow$ $P_{\cap_{n=1}^{\infty} U_{n}}\left(x_{1}\right)$, as $n \longrightarrow \infty$.

Since $x_{n+1} \in X_{n+1} \subset U_{n+1} \subset V_{n+1}$,

$$
\begin{aligned}
\bar{\phi}\left(x_{n+1}, v_{n}\right) \leq & \left(1-\alpha_{n}\right) \bar{\phi}\left(x_{n+1}, u_{n}\right)+\alpha_{n} \bar{\phi}\left(x_{n+1}, z_{n}+\varepsilon_{n}^{(1)}\right) \\
= & \left\|x_{n+1}\right\|^{2}+\left(1-\alpha_{n}\right)\left\|u_{n}\right\|^{2}-2\left(1-\alpha_{n}\right)\left\langle x_{n+1}, J_{E^{*}} u_{n}\right\rangle+\alpha_{n}\left\|z_{n}+\varepsilon_{n}^{(1)}\right\|^{2} \\
& -2 \alpha_{n}\left\langle x_{n+1}, J_{E^{*}}\left(z_{n}+\varepsilon_{n}^{(1)}\right)\right\rangle \\
= & \left\|x_{n+1}\right\|^{2}-\left(1-\alpha_{n}\right)\left\|u_{n}\right\|^{2}-\alpha_{n}\left\|z_{n}+\varepsilon_{n}^{(1)}\right\|^{2}+2\left(1-\alpha_{n}\right)\left\langle u_{n}-x_{n+1}, J_{E^{*}} u_{n}\right\rangle \\
& +2 \alpha_{n}\left\langle z_{n}+\varepsilon_{n}^{(1)}-x_{n+1}, J_{E^{*}}\left(z_{n}+\varepsilon_{n}^{(1)}\right)\right\rangle \\
\leq & \left(\left\|x_{n+1}\right\|^{2}-\left\|z_{n}+\varepsilon_{n}^{(1)}\right\|^{2}\right)+\left(1-\alpha_{n}\right)\left(\left\|z_{n}+\varepsilon_{n}^{(1)}\right\|^{2}-\left\|u_{n}\right\|^{2}\right) \\
& +2\left(1-\alpha_{n}\right)\left\|u_{n}\right\|\left\|x_{n+1}-u_{n}\right\|+2 \alpha_{n}\left\|z_{n}+\varepsilon_{n}^{(1)}\right\|\left\|z_{n}+\varepsilon_{n}^{(1)}-x_{n+1}\right\| .
\end{aligned}
$$

Since $\varepsilon_{n}^{(1)} \longrightarrow 0$, it follows from Step 5 and Lemma 2 that $\quad x_{n+1}-v_{n} \longrightarrow 0$, as $n \longrightarrow \infty$. Therefore, $v_{n} \longrightarrow P_{\cap_{n=1}^{\infty} U_{n}}\left(x_{1}\right)$, as $n \longrightarrow \infty$.

Since $x_{n+1} \in X_{n+1} \subset U_{n+1}$,

$$
\begin{aligned}
& \beta_{n}\left[\bar{\phi}\left(x_{n+1}, u_{n}\right)-\bar{\phi}\left(x_{n+1}, y_{n}+\varepsilon_{n}^{(2)}\right)\right] \\
& \leq \bar{\phi}\left(x_{n+1}, u_{n}\right)-\bar{\phi}\left(x_{n+1}, w_{n}\right) \longrightarrow 0 .
\end{aligned}
$$

Since $\beta_{n} \rightarrow 0$, there exists a subsequence of $\{n\}$, which is still denoted by $\{n\}$ such that $\bar{\phi}\left(x_{n+1}, u_{n}\right)-$ $\bar{\phi}\left(x_{n+1}, y_{n}+\varepsilon_{n}^{(2)}\right) \longrightarrow 0, \quad$ and then $x_{n+1}-$ $\left(y_{n}+\varepsilon_{n}^{(2)}\right) \longrightarrow 0$, which ensures that $y_{n} \longrightarrow P_{\cap_{n=1}^{\infty} U_{n}}\left(x_{1}\right)$, as $n \longrightarrow \infty$.

Step 7. $\quad P_{\cap_{n=1}^{\infty} U_{n}}\left(x_{1}\right) \in\left(\cap_{i=1}^{\infty}\left(T_{i} J_{E^{*}}\right)^{-1} 0\right) \cap\left(\cap_{i=1}^{\infty}\right.$ $\left.\left(S_{i} J_{E^{*}}\right)^{-1} 0\right)$.

$\forall q \in\left(\cap_{i=1}^{\infty}\left(T_{i} J_{E^{*}}\right)^{-1} 0\right) \cap\left(\cap_{i=1}^{\infty}\left(S_{i} J_{E^{*}}\right)^{-1} 0\right)$, and using Lemma 5, we have

$$
\begin{aligned}
\bar{\phi}\left(q, \widetilde{U_{n}}\left(z_{n}+\varepsilon_{n}^{(1)}\right)\right) & \leq a_{0} \bar{\phi}\left(q, z_{n}+\varepsilon_{n}^{(1)}\right)+\sum_{i=1}^{\infty} a_{n, i} \bar{\phi}\left(q,\left(J_{E^{*}}+r_{n, i} T_{i} J_{E^{*}}\right)^{-1} J_{E^{*}}\left(z_{n}+\varepsilon_{n}^{(1)}\right)\right) \\
& \leq a_{0} \bar{\phi}\left(q, z_{n}+\varepsilon_{n}^{(1)}\right)+\sum_{i=1}^{\infty} a_{n, i}\left[\bar{\phi}\left(q, z_{n}+\varepsilon_{n}^{(1)}\right)-\bar{\phi}\left(\left(J_{E^{*}}+r_{n, i} T_{i} J_{E^{*}}\right)^{-1} J_{E^{*}}\left(z_{n}+\varepsilon_{n}^{(1)}\right), u_{n}+\varepsilon_{n}^{(1)}\right)\right] \\
& =\bar{\phi}\left(q, z_{n}+\varepsilon_{n}^{(1)}\right)-\sum_{i=1}^{\infty} a_{n, i} \bar{\phi}\left(\left(J_{E^{*}}+r_{n, i} T_{i} J_{E^{*}}\right)^{-1} J_{E^{*}}\left(z_{n}+\varepsilon_{n}^{(1)}\right), z_{n}+\varepsilon_{n}^{(1)}\right) .
\end{aligned}
$$


From iterative scheme (15), we know that

$$
\begin{aligned}
\bar{\phi}\left(q, v_{n}\right) & \leq\left(1-\alpha_{n}\right) \bar{\phi}\left(q, u_{n}\right)+\alpha_{n} \bar{\phi}\left(q, \widetilde{U_{n}}\left(z_{n}+\varepsilon_{n}^{(1)}\right)\right) \\
& \leq\left(1-\alpha_{n}\right) \bar{\phi}\left(q, u_{n}\right)+\alpha_{n} \bar{\phi}\left(q, z_{n}+\varepsilon_{n}^{(1)}\right)-\alpha_{n} \sum_{i=1}^{\infty} a_{n, i} \bar{\phi}\left(\left(J_{E^{*}}+r_{n, i} T_{i} J_{E^{*}}\right)^{-1} J_{E^{*}}\left(u_{n}+\varepsilon_{n}^{(1)}\right), z_{n}+\varepsilon_{n}^{(1)}\right)
\end{aligned}
$$

which implies that

$$
\begin{aligned}
& \alpha_{n} \sum_{i=1}^{\infty} a_{n, i} \bar{\phi}\left(\left(J_{E^{*}}+r_{n, i} T_{i} J_{E^{*}}\right)^{-1} J_{E^{*}}\left(z_{n}+\varepsilon_{n}^{(1)}\right), z_{n}+\varepsilon_{n}^{(1)}\right) \\
& \leq\left(1-\alpha_{n}\right) \bar{\phi}\left(q, u_{n}\right)+\alpha_{n} \bar{\phi}\left(q, z_{n}+\varepsilon_{n}^{(1)}\right)-\bar{\phi}\left(q, v_{n}\right) \\
& \leq\left(1-\alpha_{n}\right)\left\|u_{n}\right\|^{2}+\alpha_{n}\left\|z_{n}+\varepsilon_{n}^{(1)}\right\|^{2}-\left\|v_{n}\right\|^{2}-2\left(1-\alpha_{n}\right)\left\langle q, J_{E^{*}} u_{n}\right\rangle-2 \alpha_{n}\left\langle q, J_{E^{*}}\left(z_{n}+\varepsilon_{n}^{(1)}\right)\right\rangle+2\left\langle q, J_{E^{*}} v_{n}\right\rangle \\
& \leq\left(\left\|u_{n}\right\|^{2}-\left\|v_{n}\right\|^{2}\right)+\alpha_{n}\left(\left\|z_{n}+\varepsilon_{n}^{(1)}\right\|^{2}-\left\|u_{n}\right\|^{2}\right)+2\|q\|\left\|J_{E^{*}}\left(z_{n}+\varepsilon_{n}^{(1)}\right)-J_{E^{*}} u_{n}\right\|+2\|q\|\left\|J_{E^{*}} u_{n}-J_{E^{*}} v_{n}\right\| \longrightarrow 0
\end{aligned}
$$

Since $\alpha_{n} \rightarrow 0$, there exists a subsequence of $\{n\}$, which is still denoted by $\{n\}$ such that $\bar{\phi}\left(\left(J_{E^{*}}+r_{n, i} T_{i} J_{E^{*}}\right)^{-1}\right.$ $\left.J_{E^{*}}\left(z_{n}+\varepsilon_{\eta}^{(1)}\right), z_{n}+\varepsilon_{n}^{(1)}\right) \longrightarrow 0$. Then ${ }^{(1)} \quad\left(J_{E^{*}}+\right.$ $\left.r_{n, i} T_{i} J_{E^{*}}\right)^{-1} J_{E^{*}}\left(u_{n}+\varepsilon_{n}^{(1)}\right) \longrightarrow P_{\cap_{n=1}^{\infty} V_{n}}\left(x_{1}\right), \quad$ as $n \longrightarrow \infty$.

$$
\begin{aligned}
\bar{\phi}\left(q, \widetilde{\widetilde{U}_{n}}\left(y_{n}+\varepsilon_{n}^{(2)}\right)\right) \leq & b_{0} \bar{\phi}\left(q, y_{n}+\varepsilon_{n}^{(2)}\right) \\
& +\sum_{i=1}^{\infty} b_{n, i} \bar{\phi}\left(q,\left[\left(J_{E^{*}}+t_{n, i} S_{i} J_{E^{*}}\right)^{-1} J_{E^{*}}\right]\left[\left(J_{E^{*}}+t_{n, i-1} S_{i-1} J_{E^{*}}\right)^{-1} J_{E^{*}}\right] \ldots\left[\left(J_{E^{*}}+t_{n, 1} S_{1} J_{E^{*}}\right)^{-1} J_{E^{*}}\right]\left(y_{n}+\varepsilon_{n}^{(2)}\right)\right) \\
\leq & b_{0} \bar{\phi}\left(q, y_{n}+\varepsilon_{n}^{(2)}\right) \\
& +\sum_{i=1}^{\infty} b_{n, i} \bar{\phi}\left(q,\left[\left(J_{E^{*}}+t_{n, i-1} S_{i-1} J_{E^{*}}\right)^{-1} J_{E^{*}}\right] \ldots\left[\left(J_{E^{*}}+t_{n, 1} S_{1} J_{E^{*}}\right)^{-1} J_{E^{*}}\right]\left(y_{n}+\varepsilon_{n}^{(2)}\right)\right) \\
& -\sum_{i=1}^{\infty} b_{n, i} \bar{\phi}\left(\left[\left(J_{E^{*}}+t_{n, i} S_{i} J_{E^{*}}\right)^{-1} J_{E^{*}}\right] \ldots\left[\left(J_{E^{*}}+t_{n, 1} S_{1} J_{E^{*}}\right)^{-1} J_{E^{*}}\right]\left(y_{n}+\varepsilon_{n}^{(2)}\right),\right. \\
& \left.\cdot\left[\left(J_{E^{*}}+t_{n, i-1} S_{i-1} J_{E^{*}}\right)^{-1} J_{E^{*}}\right] \ldots\left[\left(J_{E^{*}}+t_{n, 1} S_{1} J_{E^{*}}\right)^{-1} J_{E^{*}}\right]\left(y_{n}+\varepsilon_{n}^{(2)}\right)\right) .
\end{aligned}
$$

From iterative scheme (15), we have 


$$
\begin{aligned}
\bar{\phi}\left(q, w_{n}\right) \leq & \left(1-\beta_{n}\right) \bar{\phi}\left(q, u_{n}\right)+\beta_{n} \bar{\phi}\left(q, \widetilde{\widetilde{U_{n}}}\left(y_{n}+\varepsilon_{n}^{(2)}\right)\right) \\
\leq & \left(1-\beta_{n}\right) \bar{\phi}\left(q, u_{n}\right)+\beta_{n} b_{0} \bar{\phi}\left(q, y_{n}+\varepsilon_{n}^{(2)}\right) \\
& +\beta_{n}\left(\sum_{i=1}^{\infty} b_{n, i} \phi\left(q,\left(J_{E^{*}}+t_{n, i-1} S_{i-1} J_{E^{*}}\right)^{-1} J_{E^{*}}\right)\left(y_{n}+\varepsilon_{n}^{(2)}\right)\right. \\
& -\beta_{n} \sum_{i=1}^{\infty} b_{n, i} \bar{\phi}\left(\left(J_{E^{*}}+t_{n, i} S_{i} J_{E^{*}}\right)^{-1} J_{E^{*}}\right) \cdots\left(\left(J_{E^{*}}+t_{n, 1} S_{1} J_{E^{*}}\right)^{-1} J_{E^{*}}\right)\left(y_{n}+\varepsilon_{n}^{(2)}\right), \\
& \left.\cdot\left(\left(J_{E^{*}}+t_{n, i-1} S_{i-1} J_{E^{*}}\right)^{-1} J_{E^{*}}\right) \cdots\left(\left(J_{E^{*}}+t_{n, 1} S_{1} J_{E^{*}}\right)^{-1} J_{E^{*}}\right)\left(y_{n}+\varepsilon_{n}^{(2)}\right)\right) .
\end{aligned}
$$

Therefore,

$$
\begin{aligned}
\beta_{n} \sum_{i=1}^{\infty} b_{n, i}\left[\bar{\phi}\left(\left(J_{E^{*}}+t_{n, i} S_{i} J_{E^{*}}\right)^{-1} J_{E^{*}}\right) \cdots\left(\left(J_{E^{*}}+t_{n, 1} S_{1} J_{E^{*}}\right)^{-1} J_{E^{*}}\right)\left(y_{n}+\varepsilon_{n}^{(2)}\right),\right. & \left.\left(\left(J_{E^{*}}+t_{n, i-1} S_{i-1} J_{E^{*}}\right)^{-1} J_{E^{*}}\right) \cdots\left(\left(J_{E^{*}}+t_{n, 1} S_{1} J_{E^{*}}\right)^{-1} J_{E^{*}}\right)\left(y_{n}+\varepsilon_{n}^{(2)}\right)\right] \\
\leq & \left(1-\beta_{n}\right) \bar{\phi}\left(q, u_{n}\right)+\beta_{n} b_{0} \bar{\phi}\left(q, y_{n}+\varepsilon_{n}^{(2)}\right)+\beta_{n} \sum_{i=1}^{\infty} b_{n, i} \bar{\phi}\left(q, y_{n}+\varepsilon_{n}^{(2)}\right)-\bar{\phi}\left(q, w_{n}\right) \\
\leq & \left(1-\beta_{n}\right)\left\|u_{n}\right\|^{2}+\beta_{n}\left\|y_{n}+\varepsilon_{n}^{(2)}\right\|^{2}-\left\|w_{n}\right\|^{2}+2\|q\|\left\|y_{n}+\varepsilon_{n}^{(2)}-u_{n}\right\|+2\|q\|\left\|u_{n}-w_{n}\right\| \longrightarrow 0 .
\end{aligned}
$$

Since $\beta_{n} \nrightarrow 0$, there exists a subsequence of $\{n\}$, which is still denoted by $\{n\}$ such that

$$
\begin{aligned}
& \left(\left(J_{E^{*}}+t_{n, i} S_{i} J_{E^{*}}\right)^{-1} J_{E^{*}}\right) \cdots\left(\left(J_{E^{*}}+t_{n, 1} S_{1} J_{E^{*}}\right)^{-1} J_{E^{*}}\right)\left(y_{n}+\varepsilon_{n}^{(2)}\right) \\
& -\left(\left(J_{E^{*}}+t_{n, i-1} S_{i-1} J_{E^{*}}\right)^{-1} J_{E^{*}}\right) \cdots\left(\left(J_{E^{*}}+t_{n, 1} S_{1} J_{E^{*}}\right)^{-1} J_{E^{*}}\right)\left(y_{n}+\varepsilon_{n}^{(2)}\right) \longrightarrow 0 .
\end{aligned}
$$

Repeating the above process,

$$
\begin{aligned}
& \left(\left(J_{E^{*}}+t_{n, i-1} S_{i-1} J_{E^{*}}\right)^{-1} J_{E^{*}}\right) \cdots\left(\left(J_{E^{*}}+t_{n, 1} S_{1} J_{E^{*}}\right)^{-1} J_{E^{*}}\right)\left(y_{n}+\varepsilon_{n}^{(2)}\right) \\
& \left(\left(J_{E^{*}}+t_{n, i-1} S_{i-2} J_{E^{*}}\right)^{-1} J_{E^{*}}\right) \cdots\left(\left(J_{E^{*}}+t_{n, 1} S_{1} J_{E^{*}}\right)^{-1} J_{E^{*}}\right)\left(y_{n}+\varepsilon_{n}^{(2)}\right) \longrightarrow 0, \\
& \left(\left(J_{E^{*}}+t_{n, i-1} S_{i-2} J_{E^{*}}\right)^{-1} J_{E^{*}}\right) \cdots\left(\left(J_{E^{*}}+t_{n, 1} S_{1} J_{E^{*}}\right)^{-1} J_{E^{*}}\right)\left(y_{n}+\varepsilon_{n}^{(2)}\right) \\
& -\left(\left(J_{E^{*}}+t_{n, i-1} S_{i-3} J_{E^{*}}\right)^{-1} J_{E^{*}}\right) \cdots\left(\left(J_{E^{*}}+t_{n, 1} S_{1} J_{E^{*}}\right)^{-1} J_{E^{*}}\right)\left(y_{n}+\varepsilon_{n}^{(2)}\right) \longrightarrow 0, \\
& \vdots \\
& \left(\left(J_{E^{*}}+t_{n, 1} S_{1} J_{E^{*}}\right)^{-1} J_{E^{*}}\right)\left(y_{n}+\varepsilon_{n}^{(2)}\right)-\left(y_{n}+\varepsilon_{n}^{(2)}\right) \longrightarrow 0, n \longrightarrow \infty .
\end{aligned}
$$

Repeating (30), (31), and Lemma 5, one has $P_{\cap_{n=1}^{\infty} U_{n}}\left(x_{1}\right)=\left(J_{E^{*}}+t_{n, i} S \quad{ }_{i} J_{E^{*}}\right)^{-1} J_{E^{*}} \quad P_{\cap_{n=1}^{\infty} U_{n}}\left(x_{1}\right)$, $\forall i \in N$. Therefore, $J_{E^{*}} P_{\cap_{n=1}^{\infty} U_{n}}\left(x_{1}\right) \in\left(\cap_{i=1}^{\infty} T_{i}^{-1} 0\right)$ $\cap\left(\cap_{i=1}^{\infty} S_{i}^{-1} 0\right)$.
Step 8. $\overline{x_{n}}=J_{E^{*}} x_{n} \longrightarrow J_{E^{*}} P_{\cap_{n=1}^{\infty} U_{n}}\left(x_{1}\right) \in\left(\cap_{i=1}^{\infty} T_{i}^{-1} 0\right) \cap$ $\left(\cap_{i=1}^{\infty} S_{i}^{-1} 0\right)$, as $n \longrightarrow \infty$.

Using Steps 1 and $7,\left\|P_{\cap_{n=1}^{\infty} U_{n}}\left(x_{1}\right)-x_{1}\right\|=\| P$ $\left(\cap_{i=1}^{\infty}\left(T_{i} J_{E *}\right)^{-1} 0\right) \cap\left(\cap_{i=1}^{\infty}\left(S_{i} J_{E *}\right)^{-1} 0\right)\left(x_{1}\right)-x_{1} \|^{n}$. Since the metric 


$$
\begin{aligned}
& \text { projection is unique, } \\
& P_{\left(\cap_{i=1}^{\infty}\left(T_{i} J_{E *}\right)^{-1} 0\right) \cap\left(\cap_{i=1}^{\infty}\left(S_{i} J_{E *}\right)^{-1} 0\right)}\left(x_{1}\right) .
\end{aligned}
$$

This completes the proof.

Remark 1. If $\lambda_{n}=\vartheta_{n}$, then $u_{n}=z_{n}$. Two-step inertial iterative scheme (15) reduces to the traditional inertial iterative scheme.
Remark 2. If $\lambda_{n}=\vartheta_{n}=0$, then $u_{n}=z_{n}=x_{n}$; and two-step iterative scheme (15) extends the corresponding work of (13) in $[8]$.

Remark 3. If $y_{n}$ or $x_{n+1}$ is chosen as $P_{W_{n+1}} x_{1}\left(\right.$ or $\left.\pi_{W_{n+1}} x_{1}\right)$ or $P_{X_{n+1}} x_{1}$ (or $\pi_{X_{n+1}} x_{1}$ ), (15) becomes a projection iterative scheme with inertial items.

Corollary 1. In Hilbert space $H$, iterative scheme (15) becomes as follows:

$$
\left\{\begin{array}{l}
x_{0}, x_{1}, \varepsilon_{1}^{(1)}, \varepsilon_{1}^{(2)} \in H, \\
u_{n}=x_{n}+\lambda_{n}\left(x_{n}-x_{n-1}\right), \\
z_{n}=x_{n}+\vartheta_{n}\left(x_{n}-x_{n-1}\right), \\
v_{n}=\left(1-\alpha_{n}\right) u_{n}+\alpha_{n} \overline{U_{n}}\left(z_{n}+\varepsilon_{n}^{(1)}\right), \\
V_{1}=W_{1}=H, \\
V_{n+1}=\left\{p \in V_{n}: 2\left(1-\alpha_{n}\right)\left\langle p, u_{n}\right\rangle+2 \alpha_{n}\left\langle p, z_{n}+\varepsilon_{n}^{(1)}\right\rangle-2\left\langle p, v_{n}\right\rangle \leq\left(1-\alpha_{n}\right)\left\|u_{n}\right\|^{2}+\alpha_{n}\left\|z_{n}+\varepsilon_{n}^{(1)}\right\|^{2}-\left\|v_{n}\right\|^{2}\right\} \\
W_{n+1}=\left\{p \in V_{n+1}:\left\|x_{1}-p\right\|^{2} \leq\left\|P_{V_{n+1}}\left(x_{1}\right)-x_{1}\right\|^{2}+\tau_{n+1}\right\} \\
y_{n} \in W_{n+1} \text { chosen arbitrarily, } \\
w_{n}=\left(1-\beta_{n}\right) u_{n}+\beta_{n} \overline{\overline{U_{n}}}\left(y_{n}+\varepsilon_{n}^{(2)}\right), \\
U_{1}=X_{1}=H, \\
U_{n+1}=\left\{p \in V_{n+1}: 2\left(1-\beta_{n}\right)\left\langle p, u_{n}\right\rangle+2 \beta_{n}\left\langle p, y_{n}+\varepsilon_{n}^{(2)}\right\rangle-2\left\langle p, w_{n}\right\rangle \leq\left(1-\beta_{n}\right)\left\|u_{n}\right\|^{2}+\beta_{n}\left\|y_{n}+\varepsilon_{n}^{(2)}\right\|^{2}-\left\|w_{n}\right\|^{2}\right\} \\
X_{n+1}=\left\{p \in U_{n+1}:\left\|x_{1}-p\right\|^{2} \leq\left\|P_{U_{n+1}}\left(x_{1}\right)-x_{1}\right\|^{2}+\xi_{n+1}\right\}, \\
x_{n+1} \in X_{n+1} \text { chosen arbitrarily, } n \in N,
\end{array}\right.
$$

where $\overline{U_{n}}=a_{0} I+\sum_{i=1}^{\infty} a_{n, i}\left(I+r_{n, i} T_{i}\right)^{-1}$ and $\overline{\overline{U_{n}}}=b_{0} I+$ $\sum_{i=1}^{\infty} b_{n, i}\left(I+t_{n, i} S_{i}\right)^{-1} \quad\left(I+t_{n, i-1} S_{i-1}\right)^{-1} \cdots\left(I+t_{n, 1} S_{1}\right)^{-1}$, $\forall i, n \in N$. Under the assumptions of Theorem 1 , the result of Theorem 1 is still true.

\section{Numerical Experiments}

Theorem 2. Let $E=(-\infty,+\infty), T_{i} x=(x / 2 i)$, and $S_{i} x=$ $(x / i), \forall x \in(-\infty,+\infty), \forall i \in N$. Let $a_{0}=(1 / 2)=b_{0}, a_{n, i}=$ $(n+2 / 2(n+i+1)(n+i+2)), \quad b_{n, i}=\left(n / 2(n+1)^{i}\right), \quad$ and $t_{n, i}=r_{n, i}=n i, \quad \forall i, n \in N$. Let $\quad \alpha_{1}=\beta_{1}=1 ; \quad \alpha_{n}=\beta_{n}=$ $(n-1 / n),(n \geq 2) . \lambda_{n}=\vartheta_{n}=\tau_{n}=\xi_{n}=(1 / n) ; \varepsilon_{n}^{(1)}=\varepsilon_{n}^{(2)}=0$, $\forall n \in N$. For initial value $x_{0}=1, x_{1}=(1 / 3)$, the iterative sequence $\left\{x_{n}\right\}$ generated by (32) converges strongly to $0 \in\left(\cap_{i=1}^{\infty} T_{i}^{-1} 0\right) \cap\left(\cap_{i=1}^{\infty} S_{i}^{-1} 0\right)$ by the eighth step for two different choices of $\left\{y_{n}\right\}$ and $\left\{x_{n}\right\}$ in the corresponding sets $W_{n+1}$ and $X_{n+1}$, respectively.

Proof. For the special example, we can easily see that all of the assumptions of Corollary 1 are satisfied; and the iterative scheme (32) can be simplified as follows: 


$$
\left\{\begin{array}{l}
x_{0}=1, \\
x_{1}=\frac{1}{3}, \\
V_{2}=V_{1}=(-\infty,+\infty)=W_{1}, \\
U_{1}=(-\infty,+\infty)=X_{1}, \\
u_{n}=\frac{n+1}{n} x_{n}-\frac{1}{n} x_{n-1}, \\
v_{n}=\frac{n+5}{2 n+4} u_{n}, \\
V_{n+1}=V_{n} \cap\left\{p: 2\left(u_{n}-v_{n}\right) p \leq\left(u_{n}-v_{n}\right)\left(u_{n}+v_{n}\right)\right\} \\
W_{n+1}=V_{n+1} \cap\left[x_{1}-\sqrt{\left(P_{V_{n+1}} x_{1}-x_{1}\right)^{2}+\frac{1}{n+1}}, x_{1}+\sqrt{\left(P_{V_{n+1}} x_{1}-x_{1}\right)^{2}+\frac{1}{n+1}}\right. \\
y_{n} \in W_{n+1}, \\
W_{n+1}=V_{n+1} \cap\left\{p: 2\left(\frac{u_{n}}{n}+\frac{n-1}{n} y_{n}-w_{n}\right) p \leq \frac{u_{n}^{2}}{n}+\frac{n-1}{n} y_{n}^{2}-w_{n}^{2}\right\} \\
w_{n}=\frac{(1 / n) u_{n}^{2}+(n-1 / n) y_{n}^{2}-w_{n}^{2}}{2\left((1 / n) u_{n}+(n-1 / n) y_{n}-w_{n}\right)} \\
x_{n+1} \in X_{n+1}, \quad n \in N .
\end{array},\right.
$$

Now, compute step by step and choose two different groups of values of $y_{n}$ and $x_{n}$ in $W_{n+1}$ and $X_{n+1}$, respectively; we can get the two following tables.

Remark 4. From Tables 1 and 2 derived from the numerical experiments done in Theorem 2, we may find that (1) $W_{n+1}$ is an interval that permits us to choose intermediate iterative element $\left\{y_{n}\right\}$ flexibly; (2) two extreme values of $\left\{y_{n}\right\}$ in $W_{n+1}$, the largest and the smallest, are chosen, from which we can see that the convergence of the iterative sequence $\left\{x_{n}\right\}$ is not affected.

\section{Curvature Systems}

To emphasize the importance of d-accretive mappings, the connection among d-accretive mappings, iterative schemes, and nonlinear boundary value problems is set up.
Definition 7 (see [22]). A single-valued mapping $A: D(A)=$ $E \longrightarrow E^{*}$ is hemicontinuous if $A(x+t y) \rightarrow A x$, as $t \longrightarrow 0$, $\forall x, y \in E$.

Definition 8 (see $[22,23]$ ). A multivalued mapping $A: D(A) \subset E \longrightarrow 2^{E^{*}}$ is monotone if $\langle x-y, u-v\rangle \geq 0$, $\forall x, y \in D(A), u \in A x$, and $v \in A y$. The monotone operator $A$ is called maximally monotone if $R\left(J_{E}+\lambda A\right)=E^{*}, \forall \lambda>0$.

Lemma 7 (see [22]). If $A: D(A)=E \longrightarrow E^{*}$ is everywhere defined, monotone, and hemicontinuous, then it is maximally monotone.

Example 1. We shall investigate the following curvature systems: 


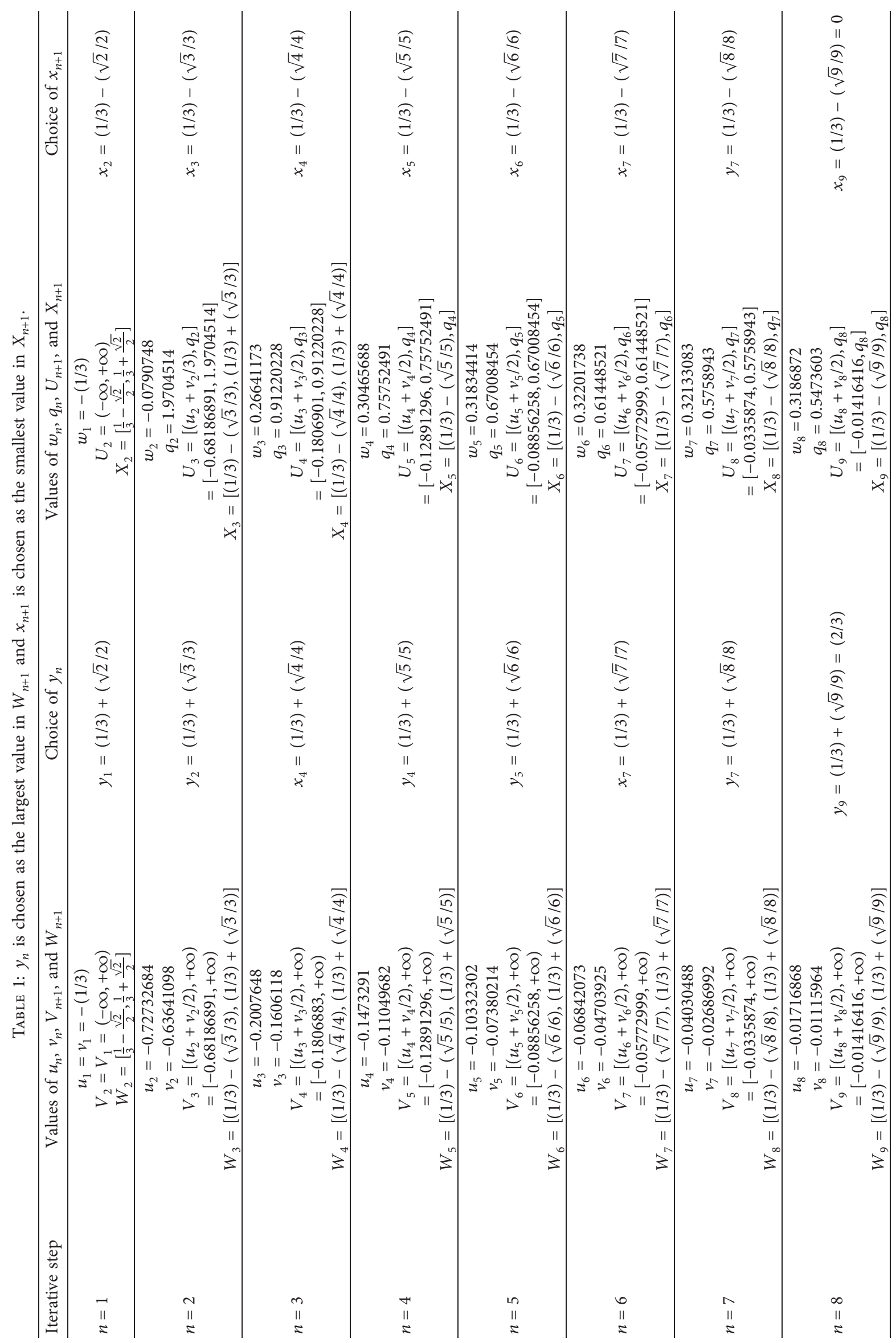




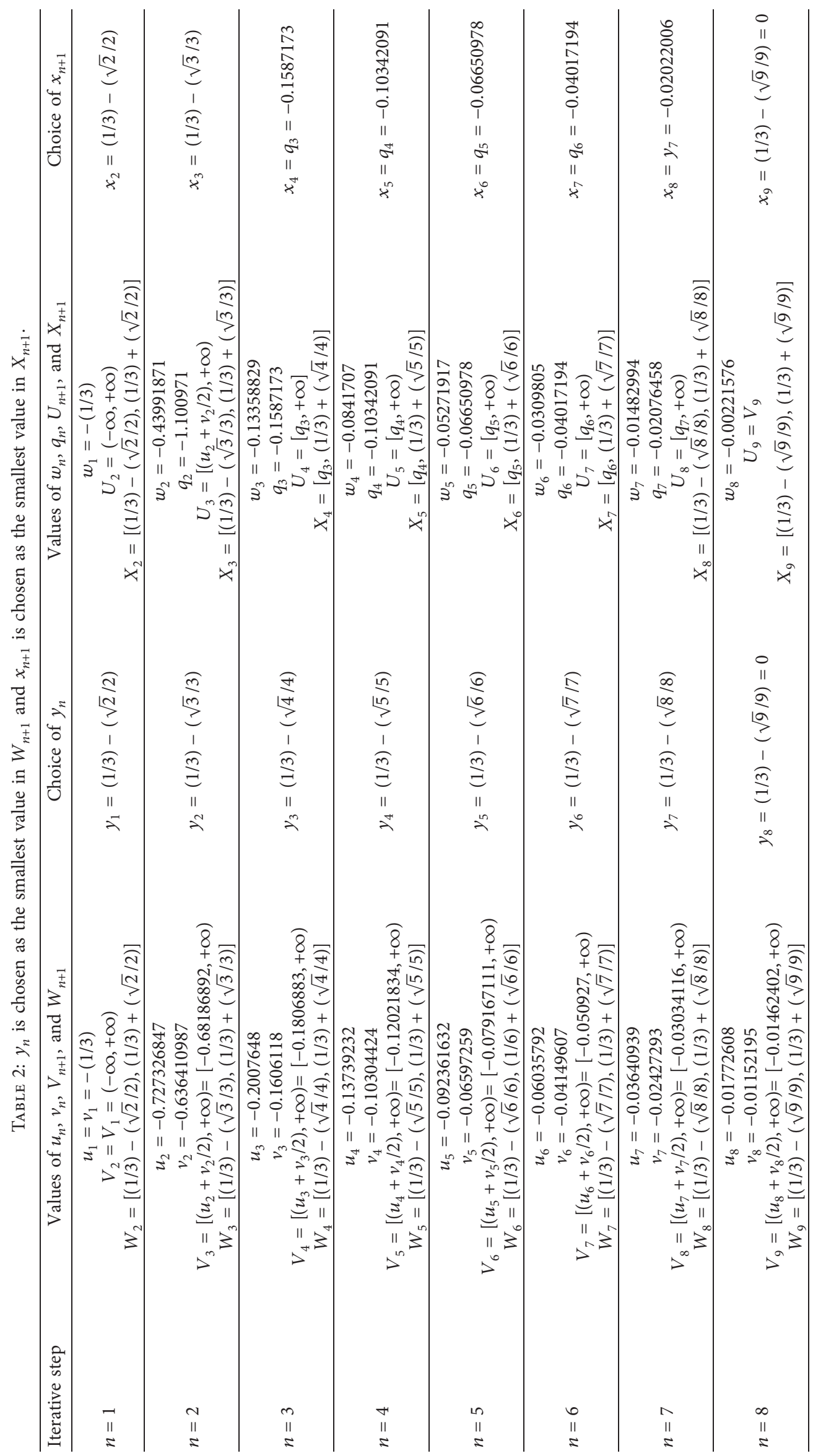


14

Journal of Mathematics

$$
\left\{\begin{array}{l}
-\operatorname{div}\left[\left(1+\left|\nabla u^{(i)}\right|^{2}\right)^{\left(s_{i} / 2\right)}\left|\nabla u^{(i)}\right|^{m_{i}-1} \nabla u^{(i)}\right]+\varepsilon\left|u^{(i)}\right|^{r_{i}-2} u^{(i)}+u^{(i)}(x)=h(x), \quad x \in \Omega, \\
-<v,\left(1+\left|\nabla u^{(i)}\right|^{2}\right)^{\left(s_{i} / 2\right)}\left|\nabla u^{(i)}\right|^{m_{i}-1} \nabla u^{(i)}>=0, \quad x \in \Gamma, i \in N,
\end{array}\right.
$$

where $|\cdot|$ and $\langle\cdot, \cdot\rangle$ denote the norm and inner-product in $R^{n}$, respectively. $\Omega$ is the bounded conical domain of $R^{n}(n \geq 1)$ with its boundary $\Gamma \in C^{1}, v$ is the normal deprivative of $\Gamma, \nabla u^{(i)}=\left(\left(\partial u^{(i)} / \partial x_{1}\right), \ldots,\left(\partial u^{(i)} / \partial x_{n}\right)\right), \epsilon$ is a nonnegative constant, and $h(x)$ is a given function. For $i \in N, m_{i}+s_{i}+1=q_{i}, \quad m_{i} \geq 0$, and $(2 n / n+1)<q_{i} \leq 2$. If $q_{i} \geq n$, then suppose that $1 \leq r_{i}<+\infty$; if $q_{i}<n$, then suppose that $1 \leq r_{i} \leq\left(n q_{i} / n-q_{i}\right)$. We use $\|\cdot\|_{q_{i}^{\prime}}$ and $\|\cdot\|_{1, q_{i}, \Omega}$ to denote the norm in $L^{q^{\prime \prime}}(\Omega)$ and $W^{1, q_{i}}(\Omega)$, respectively, $\forall i \in N$.

Lemma 8. For $i \in N$, define $B_{i}: W^{1, q_{i \prime}}(\Omega) \longrightarrow\left(W^{1, q_{i \prime}}(\Omega)\right)^{*}$ as follows: $\forall u, v \in W^{1, q_{i} I}(\Omega)$,

$$
\left\langle v, B_{i} u\right\rangle=\int_{\Omega}\left\langle\left(1+\left|\nabla\left(|u|^{q_{i j}-1} \operatorname{sgn} u\|u\|_{q_{i}}^{2-q_{i}^{\prime}}\right)\right|^{2}\right)^{\left(s_{i} / 2\right)}\left|\nabla\left(|u|^{q_{i \prime}-1} \operatorname{sgn} u\|u\|_{q_{i}^{\prime}}^{2-q_{i}}\right)\right|^{m_{i}-1} \nabla\left(|u|^{q_{i \prime}-1} \operatorname{sgn} u\|u\|_{q_{i}^{\prime}}^{2-q_{i}^{\prime}}\right) \nabla\left(|v|^{q_{i}{ }^{\prime}-1} \operatorname{sgn} v\|v\|_{q_{i}^{\prime}}^{2-\dot{q}_{i}}\right)\right\rangle \mathrm{d} x
$$

Then, $B_{i}$ is everywhere defined, hemicontinuous, and monotone, $\forall i \in N$.
Step $1 . B_{i}$ is everywhere defined.

If $s_{i} \geq 0$, then

Proof. The proof is split into three steps.

$$
\begin{aligned}
& \left|\left\langle v, B_{i} u\right\rangle\right| \leq \int_{\Omega}\left|2 \max \left\{1,\left|\nabla\left(|u|^{q_{i i}-1} \operatorname{sgn} u\|u\|_{\dot{q}_{i}^{\prime}}^{2-q_{i}^{\prime}}\right)\right|^{2}\right\}^{\left(s_{i} / 2\right)}\right|\left|\nabla\left(|u|^{q_{i} \prime^{-1}} \operatorname{sgn} u\|u\|_{\dot{q}_{i}^{\prime}}^{2-q_{i}^{\prime}}\right)\right|^{m_{i}}\left|\nabla\left(|v|^{q_{i i}{ }^{\prime}-1} \operatorname{sgn} v\|v\|_{\dot{q}_{i}^{\prime}}^{2-\dot{q}_{i}^{\prime}}\right)\right| \mathrm{d} x \\
& \leq 2^{\left(s_{i} / 2\right)}\left(\int_{\Omega} \mid \nabla\left(|u|^{q_{i \prime}-1} \operatorname{sgn} u\|u\|_{\dot{q}_{i}^{\prime}}^{2-\dot{q}_{i}^{\prime}}\right)^{m_{i} q_{i \prime}} \mathrm{d} x\right)^{\left(1 / \dot{q}_{i}^{\prime}\right)}\left(\left|\nabla\left(|v|^{q_{i \prime}-1} \operatorname{sgn} v\|v\|_{q_{i}^{\prime}}^{2-q_{i}^{\prime}}\right)\right|^{q_{i}}\right)^{\left(1 / q_{i}^{\prime}\right)} \\
& +2^{\left(s_{i} / 2\right)}\left(\int_{\Omega}\left|\nabla\left(|u|^{q_{i}{ }^{-1}} \operatorname{sgn} u\|u\|_{q^{\prime}}^{2-q_{i}^{\prime}}\right)\right|^{q_{i} \prime} \mathrm{d} x\right)^{\left(1 / q_{i}^{\prime}\right)}\left(\left|\nabla\left(|v|^{q_{i}{ }^{-1}} \operatorname{sgn} v\|v\|_{\dot{q}_{i}^{\prime}}^{2-q_{i}^{\prime}}\right)\right|^{q_{i}}\right)^{\left(1 / q_{i}^{\prime}\right)} \\
& \leq 2^{\left(s_{i} / 2\right)}\left\|\left|\nabla\left(|u|^{q_{i_{i}-1}} \operatorname{sgn} u\|u\|_{\dot{q}_{i}^{\prime}}^{2-q_{i}^{\prime}}\right)\right|\right\|_{\dot{q}_{i}^{\prime}}^{m_{i}}\left\|\left|\nabla\left(|v|^{q_{i}{ }^{\prime}-1} \operatorname{sgn} v\|v\|_{\dot{q}_{i}^{\prime}}^{2-q_{i}^{\prime}}\right)\right|\right\|_{1, \dot{q}_{i}^{\prime} \Omega}
\end{aligned}
$$

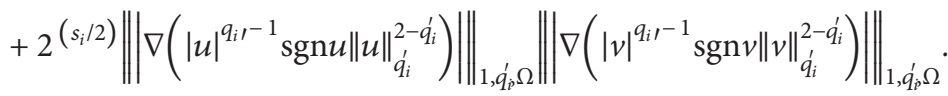

If $s_{i}<0$, then

$$
\begin{aligned}
& \left|\left\langle v, B_{i} u\right\rangle\right| \leq \int_{\Omega}\left|\nabla\left(|u|^{q_{i_{i}-1}} \operatorname{sgn} u\|u\|_{q_{i}^{\prime}}^{2-q_{i}^{\prime}}\right)\right|^{m_{i}+s_{i}}\left|\nabla\left(|v|^{q_{i}{ }^{\prime}-1} \operatorname{sgn} v\|v\|_{q_{i}^{\prime}}^{2-q_{i}^{\prime}}\right)\right| \mathrm{d} x \\
& \leq\left\|\left|\nabla\left(|u|^{q_{i \prime}-1} \operatorname{sgn} u\|u\|_{q_{i}^{\prime}}^{2-q_{i}^{\prime}}\right)\right|\right\|_{q_{i}^{\prime}}\left(q_{i}^{\prime} / q_{i}^{\prime}\right\rangle\left|\nabla\left(|v|^{q_{i}{ }^{\prime}-1} \operatorname{sgn} v\|v\|_{q_{i}^{\prime}}^{2-q_{i}^{\prime}}\right)\right| \|_{1, q_{i}^{\prime} \Omega} .
\end{aligned}
$$


Thus $B_{i}$ is everywhere defined.

For $\forall u, v \in W^{1, q_{i \prime}}(\Omega)$,

Step $2 . B_{i}$ is monotone.

$$
\begin{aligned}
& \left\langle u-v, B_{i} u-B_{i} v\right\rangle \\
& =\int_{\Omega}\left\langle\left(1+\left|\nabla\left(|u|^{\dot{q}_{i}^{\prime}-1} \operatorname{sgn} u\|u\|_{\dot{q}_{i}}^{2-\dot{q}_{i}}\right)\right|^{2}\right)^{\left(s_{i} / 2\right)}\left|\nabla\left(|u|^{\dot{q}_{i}-1} \operatorname{sgn} u\|u\|_{\dot{q}_{i}}^{2-q_{i}^{\prime}}\right)\right|^{m_{i}-1} \nabla\left(|u|^{\dot{q}_{-}-1} \operatorname{sgn} u\|u\|_{\dot{q}_{i}}^{2-\dot{q}_{i}^{\prime}}\right)\right. \\
& -\left(1+\left|\nabla\left(|v|^{\dot{q}_{-}-1} \operatorname{sgn} v\|v\|_{\dot{q}_{i}}^{2-\dot{q}_{i}}\right)\right|^{2}\right)^{\left(s_{i} / 2\right)}\left|\nabla\left(|v|^{\dot{q}_{-}-1} \operatorname{sgn} v\|v\|_{\dot{q}_{i}}^{2-\dot{q}_{i}}\right)\right|^{m_{i}-1} \nabla\left(|v|^{\dot{q}_{i}-1} \operatorname{sgn} v\|v\|_{\dot{q}_{i}}^{2-q_{i}^{\prime}}\right), \nabla\left(|u|^{\dot{q}_{i}-1} \operatorname{sgn} u\|u\|_{\dot{q}_{i}}^{2-\dot{q}_{i}}\right) \\
& \left.-\nabla\left(|v|^{q_{i}^{\prime}-1} \operatorname{sgn} v\|v\|_{q_{i}^{\prime}}^{2-q_{i}^{\prime}}\right)\right\rangle \mathrm{d} x \\
& \geq \int_{\Omega}\left[\left(1+\left|\nabla\left(|u|^{\dot{q}_{i}-1} \operatorname{sgn} u\|u\|_{\dot{q}_{i}}^{2-\dot{q}_{i}}\right)\right|^{2}\right)^{\left(s_{i} / 2\right)}\left|\nabla\left(|u|^{\dot{q}_{i}-1} \operatorname{sgn} u\|u\|_{\dot{q}_{i}^{\prime}}^{2-\dot{q}_{i}^{\prime}}\right)\right|^{m_{i}}\right. \\
& \left.-\left(1+\left|\nabla\left(|v|^{\dot{q}_{i}-1} \operatorname{sgn} v\|v\|_{\dot{q}_{i}^{\prime}}^{2-\dot{q}_{i}^{\prime}}\right)\right|^{2}\right)\left|\nabla\left(|v|^{\dot{q}_{i}-1} \operatorname{sgn} v\|v\|_{\dot{q}_{i}^{\prime}}^{2-\dot{q}_{i}^{\prime}}\right)\right|^{m_{i}}\right] \\
& \times\left(\left|\nabla\left(|u|^{\dot{q}_{i}-1} \operatorname{sgn} u\|u\|_{q_{i}^{\prime}}^{2-\dot{q}_{i}}\right)\right|-\left|\nabla\left(|v|^{\dot{q}_{i}-1} \operatorname{sgn} v\|v\|_{\dot{q}_{i}^{\prime}}^{2-\dot{q}_{i}}\right)\right|\right) \mathrm{d} x .
\end{aligned}
$$

From the fact that $h(t):=\left(1+t^{2}\right)^{\left(s_{i} / 2\right)} t^{m_{i}}, \forall t \geq 0$ is monotone; we know that $B_{i}$ is monotone.

Step 3. $B_{i}$ is hemicontinuous. $\forall u, v, w \in W^{1, q_{i \prime}}(\Omega)$ and $t \in(0,1)$; using Lebesgue's dominated convergence theorem, one has

$$
\begin{aligned}
& 0 \leq \lim _{t \longrightarrow 0}\left|\left\langle w, B_{i}(u+t v)-B_{i} u\right\rangle\right| \\
& \leq\left.\left|\int_{\Omega} \lim _{t \rightarrow 0}<\left(1+\left|\nabla\left(|u+t v|^{q_{i} / 1} \operatorname{sgn} u\|u+t v\|_{q_{i}^{\prime}}^{2-\dot{q}_{i}^{\prime}}\right)\right|^{2}\right)^{\frac{s_{i}}{2}}\right| \nabla\left(|u+t v|^{q_{i}-1} \operatorname{sgn} u\|u+t v\|_{q_{i}^{\prime}}^{2-\dot{q}_{i}^{\prime}}\right)\right|^{m_{i}-1} \\
& \times \nabla\left(|u+t v|^{q_{i \prime}-1} \operatorname{sgn}(u+t v)\|u+t v\|_{\dot{q}_{i}}^{2-q_{i}^{\prime}}\right)-\left(1+\left|\nabla\left(|u|^{q_{i \prime}-1} \operatorname{sgn} u\|u\|_{\dot{q}_{i}}^{2-q_{i}^{\prime}}\right)\right|^{2}\right)^{\frac{s_{i}}{2}}\left|\nabla\left(|u|^{q_{i i^{\prime}}-1} \operatorname{sgn} v\|u\|_{\dot{q}_{i}}^{2-q_{i}^{\prime}}\right)\right|^{m_{i}-1} \\
& \cdot \nabla\left(|u|^{q_{i i^{\prime}}-1} \operatorname{sgn} v\|u\|_{\dot{q}_{i}}^{2-q_{i}^{\prime}}\right), \nabla\left(|w|^{q_{i}{ }^{\prime}-1} \operatorname{sgn} w\|w\|_{q_{i}^{\prime}}^{2-q_{i}^{\prime}}\right)>\mathrm{d} x \mid=0 .
\end{aligned}
$$

Therefore, $B_{i}$ is hemicontinuous.

This completes the proof.

Lemma 9 (see [8]). For $i \in N$, define $C_{i}: W^{1, q_{i \prime}}(\Omega) \longrightarrow$ $\left(W^{1, q_{i \prime}}(\Omega)\right)^{*}$ as follows: $\forall u, v \in W^{1, q_{i \prime}}(\Omega)$,

$$
\left\langle v, C_{i} u\right\rangle=\int_{\Omega} u v \mathrm{~d} x
$$

Then $C_{i}$ is maximally monotone, for $\forall i \in N$.
Lemma 10 (see [22]). For each $i \in N$, there exist the maximal monotone extension of $B_{i}$ and the maximal monotone extension of $C_{i}$, which are denoted by $\overline{B_{i}}: L^{q_{i} \prime}(\Omega) \longrightarrow L^{q_{i}}(\Omega)$ and $\overline{C_{i}}: L^{q_{i l}}(\Omega) \longrightarrow L^{q_{i}}(\Omega)$, respectively.

Lemma 11 (see [24]). For $i \in N$, if $q_{i}^{\prime} \geq 2$, then the normalized duality mapping $J_{i} ; L^{q_{i \prime}}(\Omega) \longrightarrow L^{q_{i}}(\Omega)$ is defined by $J_{i} u=|u|^{q_{i}-1} \operatorname{sgn} u\|u\|_{q_{i}}^{2-q_{i}}, \quad \forall u \in L^{q_{i 1}}(\Omega)$. Then, $J_{i}^{-1}$ : $L^{q_{i}}(\Omega) \longrightarrow L^{q_{i} \prime}(\Omega)$ is ${ }^{q_{i}}$ defined by $J_{i}^{-1} u=|u|^{q_{i}{ }^{-1}} \operatorname{sgn} u$, $\forall u \in L^{q_{i}}(\Omega)$. 
Based on the above lemmas and imitating Theorems $3.10,3.11$, and 3.12 in [8], one has the following results.

Theorem 3. For $i \in N$, define $T_{i}: L^{q_{i}}(\Omega) \longrightarrow L^{q_{i}}(\Omega)$ as follows: $\forall u \in L^{q_{i}}(\Omega), T_{i} u=\overline{B_{i}} J_{i}^{-1} u(x)$. Then $T_{i}$ is d-accretive and $R\left(I+\lambda T_{i}\right)=L^{q_{i}}(\Omega), \forall \lambda>0, i \in N$.

Theorem 4. Define the mapping $\overline{S_{i}}: L^{q_{i}}(\Omega) \longrightarrow L^{q_{i}}(\Omega)$ by $\overline{S_{i}} u=\overline{C_{i}} J_{i}^{-1} u(x), \forall u(x) \in L^{q_{i}}(\Omega)$. Then $\overline{S_{i}}$ is d-accretive and $R\left(I+\lambda \overline{S_{i}}\right)=L^{q_{i}}(\Omega), \forall \lambda>0, i \in N$.

Define the mapping $S_{i}: L^{q_{i}}(\Omega) \longrightarrow L^{q_{i}}(\Omega)$ by $S_{i} u=\overline{S_{i}} u(x)-|k|^{q_{i}-1} \operatorname{sgn} k, \forall u(x) \in L^{q_{i}}(\Omega)$, where $k$ is a constant. Then $S_{i}$ is d-accretive and $R\left(I+\lambda S_{i}\right)=L^{q_{i}}(\Omega)$, $\forall \lambda>0, i \in N$.

Theorem 5. If, in (34), $f_{i}(x) \equiv \varepsilon|k|^{r_{i}-1} \operatorname{sgn} k+k$, where $k$ is a constant, then $\left\{u^{(i)}(x) \equiv k\right\}$ is the solution of (34). Moreover, $\left\{u^{(i)}(x) \equiv k\right\} \subset\left(\cap_{i=1}^{\infty} T_{i}^{-1} 0\right) \cap\left(\cap_{i=1}^{\infty} S_{i}^{-1} 0\right)$.

Proof. It is obvious that $\left\{u^{(i)}(x) \equiv k\right\}$ is the solution of (34). If $u^{(i)}(x) \equiv k$, then $T_{i} u^{(i)}(x)=\overline{B_{i}} J_{i}^{-1} k=B_{i} J_{i}^{-1} k=0$ and $S_{i} u^{(i)}(x)=\overline{C_{i}} J_{i}^{-1} k-|k|^{q_{i}-1} \operatorname{sgn} k=C_{i} J_{i}^{-1} k-|k|^{q_{i}-1}$ sgnk. Since $\left\langle v, C_{i} J_{i}^{-1} k-|k|^{q_{i}-1} \operatorname{sgn} k\right\rangle=\int_{\Omega}\left(J_{i}^{-1} k-|k|^{q_{i}-1} \operatorname{sgn} k\right)$ $v \mathrm{~d} x=0,\left\{u^{(i)}(x) \equiv k\right\} \subset\left(\cap_{i=1}^{\infty} T_{i}^{-1} 0\right) \cap\left(\cap_{i=1}^{\infty} S_{i}^{-1} 0\right)$.

This completes the proof.

Remark 5. From Theorem 5, we can see the relationship between the solution of curvature systems (34) and common zero points of two groups of $\mathrm{d}$-accretive mappings. This will help us to approximate the solution of curvature systems by using iterative schemes introduced in Section 2.

\section{Data Availability}

All data generated and analyzed during this study are included within this paper.

\section{Conflicts of Interest}

The authors declare that they have no conflicts of interest.

\section{Acknowledgments}

The first three authors were supported by the Natural Science Foundation of Hebei Province under Grant no. A2019207064, Science and Technology Key Project of Hebei Education Department under Grant no. ZD2019073, and Key Project of Science and Research of Hebei University of Economics and Business under Grant no. 2018ZD06.

\section{References}

[1] R. P. Agarwal, D. O’Regan, and D. R. Sahu, Fixed Point Theory for Lipschitz-type Mappings with Applications, Springer, Berlin, Germany, 2008.

[2] Y. I. Alber, "Metric and generalized projection operators in Banach spaces: properties and Applications," in Theory and Applications of Nonlinear Operators of Accretive and Monotone TypeDekker, New York, NY, USA, 1996.
[3] S. Kamimura and W. Takahashi, "Strong convergence of a proximal-type algorithm in a Banach space," SIAM Journal on Optimization, vol. 13, pp. 938-945, 2003.

[4] U. Mosco, "Convergence of convex sets and of solutions of variational inequalities," Advances in Mathematics, vol. 3, no. 4, pp. 510-585, 1969.

[5] M. Tsukada, "Convergence of best approximations in a smooth Banach space," Journal of Approximation Theory, vol. 40, no. 4, pp. 301-309, 1984.

[6] V. Barbu, Nonlinear Semigroups and Differential Equations in Banach Space, Noordhoff, Norton Shores, MI, USA, 1976.

[7] L. Wei, Y. X. Liu, and R. P. Agarwal, "Convergence theorems of convex combination methods for treating d-accretive mappings in a Banach space and nonlinear equation," Journal of Inequalities and Applications, vol. 482, 2014.

[8] L. Wei, Y. N. Zhang, and R. P. Agarwal, "A new shrinking iterative scheme for d-accretive mappings with applications to capillarity systems," Journal of Nonlinear Functional Analysis, vol. 11, 2020.

[9] L. Wei, R. P. Agarwal, and L. L. Duan, "New iterative designs for an infinitely family of d-accretive mappings in a Banach space," Journal of Nonlinear Functional Analysis, vol. 4, 2019.

[10] W. Takahashi, "Proximal point algorithms and four resolvents of nonlinear operators of monotone type in Banach spaces," Taiwanese Journal of Mathematics, vol. 12, no. 8, pp. 18831910, 2008.

[11] H.-K. Xu, "Inequalities in Banach spaces with applications," Nonlinear Analysis: Theory, Methods \& Applications, vol. 16, no. 12, pp. 1127-1138, 1991.

[12] X. Qin, S. Y. Cho, and L. Wang, "Convergence of splitting algorithms for the sum of two accretive operators with applications," Fixed Point Theory and Applications, vol. 166, 2014.

[13] X. Qin, S. Y. Cho, and L. Wang, "Strong convergence of an iterative algorithm involving nonlinear mappings of nonexpansive and accretive type," Optimization, vol. 67, no. 9, pp. 1377-1388, 2018.

[14] B. A. B. Dehaish, "Weak and strong convergence of algorithms for the sum of two accretive operators with applications," Journal of Nonlinear and Convex Analysis, vol. 16, pp. 1321-1336, 2015.

[15] X. Qin and J. C. Yao, "Weak convergence of a Mann-like algorithm for nonexpansive and accretive operators," Journal of Inequalities and Applications, vol. 232, 2017.

[16] X. Qin, L. Wang, and J. C. Yao, "Inertial splitting method for maximal monotone mappings," Journal of Nonlinear and Convex Analysis, vol. 21, pp. 2325-2333, 2020.

[17] Y. Alber and S. Reich, "Convergence of averaged approximations to null points of a class of nonlinear mapping," Communications on Applied Nonlinear Analysis, vol. 7, pp. 1-20, 2000.

[18] W. R. Guan, Construction of Iterative Algorithm for Equilibrium Points of Nonlinear Systems. Dissertation of Doctoral Degree, Ordnance Engineering College, Hebei, China, 2007.

[19] L. Wei and R. P. Agarwal, "Relaxed iterative methods for an infinite family of d-accretive mappings in a Banach space and their applications," Journal of Nonlinear Functional Analysis, vol. 16, 2018.

[20] B. T. Polyak, "Some methods of speeding up the convergence of iteration methods," USSR Computational Mathematics and Mathematical Physics, vol. 4, no. 5, pp. 1-17, 1964.

[21] D. A. Lorena and T. Pock, "An inertial forward-backward algorithm for monotone inclusions," Journal of Mathematical Imaging and Vision, vol. 51, pp. 311-325, 2015. 
[22] D. Pascali and S. Sburlan, Nonlinear Mappings of Monotone Type, Springer, Berlin, Germany, 1978.

[23] L. Wei, R. Chen, Y. N. Zhang, and R. P. Agarwal, "New shrinking iterative methods for infinite families of monotone operators in a banach space, computational experiments and applications," Journal of Inequalities and Applications, vol. 67, 2020.

[24] B. D. Calvert and C. P. Gupta, "Nonlinear elliptic boundary value problems in $L^{p}$-spaces and sums of ranges of accretive operators," Nonlinear Analysis: Theory, Methods \& Applications, vol. 2, no. 1, pp. 1-26, 1978. 